\title{
A Random Parameter Model for Continuous-Time Mean-Variance Asset-Liability Management
}

\author{
Hui-qiang Ma, ${ }^{1,2}$ Meng Wu, ${ }^{3}$ and Nan-jing Huang ${ }^{4}$ \\ ${ }^{1}$ School of Economics, Southwest University for Nationalities, Chengdu, Sichuan 610041, China \\ ${ }^{2}$ School of Finance and Statistics, Hunan University, Changsha, Hunan 410079, China \\ ${ }^{3}$ Business School, Sichuan University, Chengdu, Sichuan 610064, China \\ ${ }^{4}$ Department of Mathematics, Sichuan University, Chengdu, Sichuan 610064, China \\ Correspondence should be addressed to Meng Wu; shancherish@hotmail.com
}

Received 15 May 2015; Accepted 6 September 2015

Academic Editor: Leonid Shaikhet

Copyright (C) 2015 Hui-qiang Ma et al. This is an open access article distributed under the Creative Commons Attribution License, which permits unrestricted use, distribution, and reproduction in any medium, provided the original work is properly cited.

\begin{abstract}
We consider a continuous-time mean-variance asset-liability management problem in a market with random market parameters; that is, interest rate, appreciation rates, and volatility rates are considered to be stochastic processes. By using the theories of stochastic linear-quadratic (LQ) optimal control and backward stochastic differential equations (BSDEs), we tackle this problem and derive optimal investment strategies as well as the mean-variance efficient frontier analytically in terms of the solution of BSDEs. We find that the efficient frontier is still a parabola in a market with random parameters. Comparing with the existing results, we also find that the liability does not affect the feasibility of the mean-variance portfolio selection problem. However, in an incomplete market with random parameters, the liability can not be fully hedged.
\end{abstract}

\section{Introduction}

Mean-variance portfolio selection model was pioneered by Markowitz [1] in the single-period setting. In his seminal paper, Markowitz proposed the variance as the measure of the risk. The advantage of using variance for measuring the risk of a portfolio is due to the simplicity of computation. Thus, the mean-variance approach has inspired literally hundreds of extensions and applications and also has been commonly used in practical financial decisions. For example, Wang and Xia [2] gave an excellent review on portfolio selection problem. $\mathrm{Li}$ and $\mathrm{Ng}$ [3] employed the framework of multiobjective optimization and an embedding technique to obtain the exact mean-variance efficient frontier for multiperiod investment. $\mathrm{Wu}$ and $\mathrm{Li}[4]$ investigated a multiperiod mean-variance portfolio selection with regime switching and uncertain exit time. Zhou and Li [5] studied a continuous-time mean-variance portfolio selection problem under a stochastic LQ framework. Furthermore, Li et al. [6] considered a continuous-time mean-variance portfolio selection problem with no-shorting constraints. Under partial information, Xiong and Zhou [7] and Wang and Wu
[8] considered a continuous-time mean-variance portfolio selection problem and a problem of hedging contingent claims by portfolios, respectively.

Among several extensions of the classic mean-variance portfolio selection model, asset and liability management problem is an important subject in both academic literatures and the real world situations. In the real world, liability is so important that almost all financial institutions and individual investors should manage their debt. Thus, incorporating liability into the portfolio selection model can make investment strategies more practical. The research on mean-variance asset-liability management also evokes recent concern. Sharpe and Tint [9] first investigated meanvariance asset-liability management in a single-period setting. Leippold et al. [10] considered a multiperiod assetliability management problem and derived both the analytical optimal policy and the efficient frontier. Chiu and Li [11] studied a mean-variance asset-liability management problem in the continuous-time case where the liability was governed by a geometric Brownian motion (GBM). Xie et al. [12] also considered a continuous-time asset-liability management problem under the mean-variance criterion where the 
dynamic of liability is a Brownian motion with drift. Further, Xie [13] studied a mean-variance portfolio selection model with stochastic liability in a Markovian regime switching financial market. Zeng and $\mathrm{Li}$ [14] investigated an assetliability management problem in a jump diffusion market. Yao et al. [15] studied continuous-time mean-variance assetliability management with endogenous liabilities. By using the time-consistent approach, Wei et al. [16] considered a mean-variance asset-liability management problem with regime switching.

Among these studies, we note that all market parameters are assumed to be deterministic. However, in the real world, market parameters observed in many situations are always uncertain (see, e.g., [17-20]). In order to capture the features of optimal investment strategies with random parameters, random parameter models have drawn more attention over last few years. For example, Lim and Zhou [21] investigated a mean-variance portfolio selection problem with random parameters in a complete market and derived efficient investment strategies as well as the efficient frontier analytically in terms of the solution of BSDEs. Further, Lim [22] extended Lim and Zhou's [21] results to the case where the market is incomplete.

Up to now, the studies on the asset-liability management problem are under a common assumption that all parameters are assumed to be known with certainty. An interesting and unexplored question is what happens in a more realistic situation with random parameters. This is the main focus of our research. In view of this, we study a mean-variance assetliability management problem with random parameters and derive both the mean-variance optimal portfolio strategies and the efficient frontier. Referring to Lim [22], we consider a market where the related market parameters are random, such as interest rate, the appreciation rates, and the volatility rates of stocks' price. Further, we routinely assume that the liability is dynamically exogenous and evolves according to a Brownian motion with drift. Note that this description of liability has been widely used (see, e.g., $[12,23,24])$. Under the above assumptions, we introduce an unconstrained stochastic control problem with random parameters and derive the optimal control strategies in terms of the solutions of BSDEs. Then, by using the Lagrange multiplier technique, we derive both the mean-variance optimal investment strategies and the efficient frontier.

Our model is most closely related to the model of Lim [22]. The main differences between our model and Lim's model are in two dimensions. Firstly, we consider a portfolio selection problem with liability. Since the liability is dynamically exogenous, the driving factors of the wealth in our model include that of stocks' price and liability, which is an essential difficulty in our model but not encountered in [22]. Secondly, due to the introduction of random liability, the wealth process derived from our model is no longer homogenous with respect to the control variables, whereas the wealth process in the model without liability (see, e.g., $[21,22])$ is homogenous.

This paper proceeds as follows. In Section 2, we give some preliminaries and formulate a continuous-time meanvariance portfolio selection model with liability and random parameters. In Section 3, we introduce an unconstrained stochastic LQ control problem and derive the optimal policies and value function in closed forms in terms of the solution of BSDEs. Further, Section 4 presents the optimal investment strategies and the efficient frontier for the mean-variance asset-liability management problem with random parameters. Section 5 concludes the paper.

\section{Model Formulation}

In this section, we describe the financial market, the liability, and the mean-variance asset-liability management problem, respectively. Throughout this paper, let $T$ be a fixed terminal time, $(\Omega, \mathscr{F}, P)$ a complete probability space, and $M^{\prime}$ the transpose of the vector or matrix $M$.

2.1. The Financial Market. Let $\left(\Omega, \mathscr{F}, P,\left\{\mathscr{F}_{t}\right\}_{t \geq 0}\right)$ be a filtered complete probability space on which a standard $\left\{\mathscr{F}_{t}\right\}_{t \geq 0^{-}}$ adapted $m+d$-dimensional Brownian motion $\bar{W}(t)$ := $\left(W(t)^{\prime}, B(t)^{\prime}\right)^{\prime}:=\left(W^{1}(t), \ldots, W^{m}(t), B^{1}(t), \ldots, B^{d}(t)\right)^{\prime}$ for $m \geq 1$ and $d \geq 0$ is defined. It is assumed that $\mathscr{F}_{t}=\sigma\{\bar{W}(s)$ : $s \leq t\}$. In this paper, we use $B(t)$ to model the financial market incompleteness as $\operatorname{Lim}[22] \mathrm{did}$. When $d=0$, the financial market corresponds to a complete market.

Consider a financial market with $m+1$ securities which consists of a bond and $m$ stocks. The price of bond $A^{0}(t)$ satisfies the following differential equation:

$$
\begin{aligned}
d A^{0}(t) & =r(t) A^{0}(t) d t, \quad t \in[0, T], \\
A^{0}(0) & =A_{0}^{0}>0,
\end{aligned}
$$

where the interest rate is as follows: $r(t)>0$. The price of the $i$ th stock, $A^{i}(t)$, is described by the following stochastic differential equation (SDE):

$$
\begin{aligned}
& d A^{i}(t)=A^{i}(t)\left[\mu^{i}(t) d t+\sum_{j=1}^{m} \sigma^{i j}(t) d W^{j}(t)\right], \\
& A^{i}(0)=A_{0}^{i}>0,
\end{aligned}
$$

where $\mu^{i}(t)>0$ and $\sigma^{i}(t):=\left(\sigma^{i 1}(t), \ldots, \sigma^{i m}(t)\right)$ are appreciation rate and volatility rate of the $i$ th stock, respectively. The $\mathbb{R}^{m \times m}$-valued process of volatility coefficients

$$
\sigma(t):=\left(\sigma^{1}(t)^{\prime}, \ldots, \sigma^{m}(t)^{\prime}\right)^{\prime}
$$

is known as the volatility. In addition, we assume that the market parameters $r(\cdot), \mu^{i}(\cdot)$, and $\sigma^{i j}(\cdot)$ are $\left\{\mathscr{F}_{t}\right\}_{t \geq 0}$-adapted stochastic processes.

2.2. Liability. We assume that an exogenous accumulative liability $L(t)$ is governed by

$$
\begin{aligned}
& d L(t)=u(t) d t+v(t) d B_{L}(t), \\
& L(0)=L_{0}>0,
\end{aligned}
$$


where $B_{L}(t)$ is a one-dimensional standard Brownian motion. We assume that the diffusion term of the liability, $B_{L}$, is correlated with $\bar{W}(t)$, and $\rho=\left(\rho_{1}, \ldots, \rho_{m+d}\right)^{\prime}$ is the correlation coefficient. Then, $B_{L}(t)$ can be further expressed as follows (see (2.6) of [25] for more details):

$$
B_{L}(t)=\rho^{\prime} \bar{W}(t)+\sqrt{1-\rho^{\prime} \rho} W^{0}(t),
$$

where $W^{0}(t)$ is a standard Brownian motion which is independent of $\bar{W}(t)$. It follows from Itô's formula that

$$
d B_{L}(t)=\rho^{\prime} d \bar{W}(t)+\sqrt{1-\rho^{\prime} \rho} d W^{0}(t) .
$$

Thus, the liability $L(t)$ can be rewritten as

$$
\begin{aligned}
d L(t)= & u(t) d t-\delta_{1}(t)^{\prime} d W(t)-\delta_{2}(t)^{\prime} d B(t) \\
& -\delta_{0}(t) d W^{0}(t),
\end{aligned}
$$

where $\delta_{1}(t):=-v(t)\left(\rho_{1}, \ldots, \rho_{m}\right)^{\prime}, \delta_{2}(t):=-v(t)\left(\rho_{m+1}, \ldots\right.$, $\left.\rho_{m+d}\right)^{\prime}$, and $\delta_{0}(t):=-v(t) \sqrt{1-\rho^{\prime} \rho}$. Further, we assume that $u(\cdot)$ and $\delta(\cdot):=\left(\delta_{1}(\cdot)^{\prime}, \delta_{2}(\cdot)^{\prime}, \delta_{0}(\cdot)\right)^{\prime}$ are $\left\{\widehat{\mathscr{F}}_{t}\right\}_{t \geq 0}$-adapted stochastic processes, where $\widehat{\mathscr{F}}_{t}:=\sigma\left\{\left(\bar{W}(s)^{\prime}, W^{0}(s)\right)^{\prime}: s \leq t\right\}$.

Remark 1. When $B_{L}(t)$ is independent of $\bar{W}(t)$, that is, $\rho=0$, $B_{L}(t)$ is equal to $W^{0}(t)$. When $\rho^{\prime} \rho=1, B_{L}(t)$ can be expressed as a linear combination of $W^{1}(t), \ldots, W^{m}(t), B^{1}(t), \ldots, B^{d}(t)$.

Remark 2. Since $r(\cdot), \mu^{i}(\cdot)$, and $\sigma^{i j}(\cdot)$ are the parameters for describing the financial market and $u(\cdot)$ and $v(\cdot)$ are used to describe the exogenous liability, it is reasonable to assume that $r(\cdot), \mu^{i}(\cdot)$, and $\sigma^{i j}(\cdot)$ are $\left\{\mathscr{F}_{t}\right\}_{t \geq 0}$-adapted for $i, j=1, \ldots, m$, and $u(\cdot), v(\cdot)$, and $\delta(\cdot)$ are $\left\{\widehat{\mathscr{F}}_{t}\right\}_{t \geq 0}$-adapted.

\subsection{The Mean-Variance Asset-Liability Management Model.} Suppose that the trading of shares takes place continuously in a self-financing fashion and there are no transaction costs. We assume that an investor has an initial endowment $w$ and a liability $L(t)$. We denote by $X(t)$ the net total wealth of the investor at time $t \in[0, T]$ and by $\pi^{i}(t), i=1, \ldots, m$, the market value of the investor's wealth in the ith stock. Then, $\pi(t):=\left(\pi^{1}(t), \ldots, \pi^{m}(t)\right)^{\prime}$ is a portfolio. The net total wealth satisfies the following equation:

$$
\begin{aligned}
d X(t)= & (r(t) X(t)+b(t) \pi(t)-u(t)) d t \\
& +\left(\pi(t)^{\prime} \sigma(t)+\delta_{1}(t)^{\prime}\right) d W(t) \\
& +\delta_{2}(t)^{\prime} d B(t)+\delta_{0}(t) d W^{0}(t), \\
X(0)= & X_{0}=w-L_{0},
\end{aligned}
$$

where $b(t)=\left(\mu^{1}(t)-r(t), \ldots, \mu^{m}(t)-r(t)\right)$.

Next, we introduce the following notations.

One has $|x|:=\sqrt{\sum_{i=1}^{n} x_{i}^{2}}$, where $x=\left(x_{1}, \ldots, x_{n}\right)^{\prime} \in \mathbb{R}^{n}$.

$\mathscr{L}_{\mathscr{F}}^{2}\left(0, T ; \mathbb{R}^{n}\right)$ is the set of $\left\{\mathscr{F}_{t}\right\}_{t \geq 0}$-adapted, $\mathbb{R}^{n}$-valued stochastic processes on $[0, T]$ such that

$$
E \int_{0}^{T}|f(t)|^{2} d t<\infty .
$$

$\mathscr{L}_{\mathscr{F}}^{\infty}(\Omega ; C(0, T ; \mathbb{R}))$ is the set of $\left\{\mathscr{F}_{t}\right\}_{t \geq 0}$-adapted essentially bounded stochastic processes on $[0, T]$ with continuous sample paths.

$\mathscr{L}_{\widehat{\mathscr{F}}}^{2}\left(0, T ; \mathbb{R}^{n}\right)$ is the set of $\left\{\widehat{\mathscr{F}}_{t}\right\}_{t \geq 0}$-adapted, $\mathbb{R}^{n}$-valued stochastic processes on $[0, T]$ such that

$$
E \int_{0}^{T}|f(t)|^{2} d t<\infty .
$$

$\mathscr{L}_{\widehat{F}}^{2}(\Omega ; C(0, T ; \mathbb{R}))$ is the set of $\left\{\widehat{\mathscr{F}}_{t}\right\}_{t \geq 0}$-adapted, $\mathbb{R}$ valued stochastic processes on $[0, T]$ with $P$-a.s. continuous sample paths such that $\operatorname{Esup}_{t \in[0, T]}|f(t)|^{2}<\infty$.

$\mathscr{L}_{\overparen{\mathscr{F}}}^{2 \text {,loc }}\left(0, T ; \mathbb{R}^{n}\right)$ is the set of $\left\{\widehat{\mathscr{F}}_{t}\right\}_{t \geq 0}$-adapted, $\mathbb{R}^{n}$-valued stochastic processes on $[0, T]$ such that

$$
\int_{0}^{T}|f(t)|^{2} d t<\infty, \quad P \text {-a.s. }
$$

$\mathscr{L}_{\widehat{\mathscr{F}}_{T}}^{2}(\Omega ; \mathbb{R})$ is the set of $\widehat{\mathscr{F}}_{T}$-measurable, square-integrable random variables.

$\mathscr{L}_{\widetilde{\mathscr{F}}}^{\infty}\left(0, T ; \mathbb{R}^{m}\right)$ is the set of $\left\{\widehat{\mathscr{F}}_{t}\right\}_{t \geq 0}$-adapted essentially bounded stochastic processes on $[0, T]$.

Definition 3. A portfolio policy $\pi(\cdot)$ is said to be admissible if $\pi(\cdot) \in \mathscr{L}_{\overparen{F}}^{2}\left(0, T ; \mathbb{R}^{m}\right)$ and there exists a unique solution of $(8)$. In this case, we refer to $(X(\cdot), \pi(\cdot))$ as an admissible pair.

In this paper, we study the classical mean-variance asset-liability management problem where the liability is an exogenous liability $L(t)$. The objective of the investor is to find a portfolio $\pi(\cdot)$ to minimize his/her risk which is measured by the variance of the net terminal wealth subject to archiving a prescribed expected terminal wealth. Then, the mean-variance asset-liability management problem can be formulated as follows:

$$
\begin{array}{ll}
J^{*}:=\min & \operatorname{Var}(X(T))=E[X(T)-c]^{2}, \\
\text { subject to: } & E X(T) \\
& =c,(X(\cdot), \pi(\cdot)) \text { is admissible for }(8),
\end{array}
$$

where $c \in \mathbb{R}$ is the prescribed expected terminal wealth. It is clear that (12) is a linearly constrained convex program problem. Thus, it can be reduced to an unconstrained problem by introducing a Lagrange multiplier. Therefore, in Section 3, we first consider the following unconstrained problem parameterized by $l \in \mathbb{R}$,

$$
\min E[X(T)-l]^{2},
$$

subject to: $(X(\cdot), \pi(\cdot))$ is admissible for $(8)$,

and approach it from the perspective of stochastic LQ optimal control and BSDEs. Further, in Section 4, based on the results in Section 3, we employ the Lagrange multiplier method to derive the mean-variance efficient portfolio and the efficient frontier.

In addition, we assume that the following assumptions are satisfied throughout this paper. 
Assumption 4. Consider the following:

$$
\begin{gathered}
r(\cdot), \mu^{i}(\cdot), \sigma^{i j}(\cdot) \in \mathscr{L}_{\mathscr{F}}^{\infty}(0, T ; \mathbb{R}), \quad i, j=1, \ldots, m ; \\
u(\cdot), v(\cdot) \in \mathscr{L}_{\mathscr{F}}^{\infty}(0, T ; \mathbb{R}) ; \\
\delta(\cdot) \in \mathscr{L}_{\widetilde{F}}^{\infty}\left(0, T ; \mathbb{R}^{m+d+1}\right) ; \\
\sigma(t) \sigma(t)^{\prime} \geq \epsilon I_{m},
\end{gathered}
$$

$\forall t \in[0, T]$, for some $\epsilon>0$,

where $I_{m}$ is the $m \times m$ identity matrix. Note that $\sigma(t) \sigma(t)^{\prime} \geq$ $\epsilon I_{m}$ is the so-called nondegeneracy condition and implies that $\sigma(t)$ is invertible.

\section{The Unconstrained Asset-Liability Management Problem}

The aim of this section is to derive the optimal solution for the unconstrained problem (13).

Consider the following BSDEs:

$$
\begin{aligned}
& d p(t)=\left[\left(-2 r(t)+|\theta(t)|^{2}\right) p(t)+2 \theta(t)^{\prime} \Lambda_{1}(t)\right. \\
& \left.+\frac{1}{p(t)}\left|\Lambda_{1}(t)\right|^{2}\right] d t+\Lambda_{1}(t)^{\prime} d W(t)+\Lambda_{2}(t)^{\prime} d B(t), \\
& p(T)=1, \quad p(t)>0, \forall t \in[0, T], \\
& d h(t)=\left(r(t) h(t)+\theta(t)^{\prime} \eta_{1}(t)-\frac{\Lambda_{2}(t)^{\prime}}{p(t)} \eta_{2}(t)\right. \\
& \left.\quad-u(t)-\theta(t)^{\prime} \delta_{1}(t)+\delta_{2}(t)^{\prime} \frac{\Lambda_{2}(t)}{p(t)}\right) d t+\eta_{1}(t)^{\prime} d W(t) \\
& \quad+\eta_{2}(t)^{\prime} d B(t)+\eta_{0}(t) d W^{0}(t), \\
& h(T)=l,
\end{aligned}
$$

where $\theta(t)=\sigma(t)^{-1} b(t)^{\prime}$. Throughout this paper, a pair of processes $(p(\cdot), \Lambda(\cdot))$ is called a solution of BSDE (15) if it satisfies BSDE (15) and

$$
\begin{array}{r}
\Lambda(\cdot)=\left(\Lambda_{1}(\cdot)^{\prime}, \Lambda_{2}(\cdot)^{\prime}\right)^{\prime}, \\
(p(\cdot), \Lambda(\cdot)) \in \mathscr{L}_{\mathscr{F}}^{\infty}(\Omega ; C(0, T ; \mathbb{R})) \\
\cdot \mathscr{L}_{\mathscr{F}}^{2}\left(0, T ; \mathbb{R}^{m+d}\right), \\
\frac{1}{p(\cdot)} \in \mathscr{L}_{\mathscr{F}}^{\infty}(\Omega ; C(0, T ; \mathbb{R})) .
\end{array}
$$

On the other hand, a pair $(h(\cdot), \eta(\cdot))$ is called a solution of $\operatorname{BSDE}(16)$ if $(h(\cdot), \eta(\cdot))$ satisfies BSDE (16) and

$$
\begin{aligned}
\eta(\cdot)= & \left(\eta_{1}(\cdot)^{\prime}, \eta_{2}(\cdot)^{\prime}, \eta_{0}(\cdot)\right)^{\prime}, \\
(h(\cdot), \eta(\cdot)) \in & \mathscr{L}_{\overparen{F}}^{2}(\Omega ; C(0, T ; \mathbb{R})) \\
& \cdot \mathscr{L}_{\overparen{F}}^{2}\left(0, T ; \mathbb{R}^{m+d+1}\right) .
\end{aligned}
$$

Before deriving the optimal solution for problem (13), we will prove the existence and uniqueness of solutions of BSDEs (15) and (16), respectively. The following result can be found in [22] (see Theorem 6.1 of [22]).

Lemma 5. If Assumption 4 holds, then the following BSDE,

$$
\begin{aligned}
& d p(t)=\left[\left(-2 r(t)+|\theta(t)|^{2}\right) p(t)+2 \theta(t)^{\prime} \Lambda_{1}(t)\right. \\
& \left.+\frac{\left|\Lambda_{1}(t)\right|^{2}}{p(t)}\right] d t+\Lambda_{1}(t)^{\prime} d W(t)+\widetilde{\Lambda}_{2}(t)^{\prime} d \widetilde{B}(t), \\
& p(T)=1, \quad p(t)>0, \forall t \in[0, T],
\end{aligned}
$$

has a solution $(p(\cdot), \widetilde{\Lambda}(\cdot))$ where $\widetilde{B}(\cdot):=\left(B(\cdot)^{\prime}, W^{0}(\cdot)\right)^{\prime}$. Moreover, if $(\bar{p}(\cdot), \bar{\Lambda}(\cdot))$ and $(p(\cdot), \widetilde{\Lambda}(\cdot))$ are solutions of (19), then $\bar{p}(\cdot) \equiv p(\cdot)$.

Here, we claim that $\bar{\Lambda}(\cdot) \equiv \widetilde{\Lambda}(\cdot)$ holds. In fact, by applying Itô's formula to $\Delta p(t):=p(t)-\bar{p}(t)$, we have

$$
\begin{aligned}
d \Delta p(t)= & \Delta F(t) d t+\Delta \Lambda_{1}(t)^{\prime} d W(t) \\
& +\Delta \Lambda_{2}(t)^{\prime} d \widetilde{B}(t) \\
\Delta p(T)= & 0
\end{aligned}
$$

where $\Delta \Lambda_{1}(\cdot):=\widetilde{\Lambda}_{1}(\cdot)-\bar{\Lambda}_{1}(\cdot), \Delta \Lambda_{2}(\cdot):=\widetilde{\Lambda}_{2}(\cdot)-\bar{\Lambda}_{2}(\cdot)$, and

$$
\begin{aligned}
\Delta F(\cdot):= & \left(-2 r(\cdot)+|\theta(\cdot)|^{2}\right) \Delta p(\cdot)+2 \theta(\cdot)^{\prime} \Delta \Lambda_{1}(\cdot) \\
& +\frac{1}{p(\cdot)}\left(\left|\widetilde{\Lambda}_{1}(\cdot)\right|^{2}-\left|\bar{\Lambda}_{1}(\cdot)\right|^{2}\right) .
\end{aligned}
$$

Once again, it follows from Itô's formula that

$$
\begin{aligned}
& d(\Delta p(t))^{2} \\
& =\left(2 \Delta p(t) \Delta F(t)+\left|\Delta \Lambda_{1}(t)\right|^{2}+\left|\Delta \Lambda_{2}(t)\right|^{2}\right) d t \\
& \quad+2 \Delta p(t) \Delta \Lambda_{1}(t)^{\prime} d W(t) \\
& \quad+2 \Delta p(t) \Delta \Lambda_{2}(t)^{\prime} d \widetilde{B}(t), \\
& (\Delta p(T))^{2}=0 .
\end{aligned}
$$


Thus we have

$$
\begin{aligned}
& -E(\Delta p(t))^{2}=E \int_{t}^{T}\left(2 \Delta p(s) \Delta F(s)+\left|\Delta \Lambda_{1}(s)\right|^{2}\right. \\
& \left.+\left|\Delta \Lambda_{2}(s)\right|^{2}\right) d s .
\end{aligned}
$$

From Lemma 5, we know that $\Delta p(\cdot) \equiv 0$ and so

$$
0=E \int_{t}^{T}\left(\left|\Delta \Lambda_{1}(s)\right|^{2}+\left|\Delta \Lambda_{2}(s)\right|^{2}\right) d s
$$

This implies that $\bar{\Lambda}(\cdot) \equiv \widetilde{\Lambda}(\cdot)$ holds.

Since $r(\cdot)$ and $\theta(\cdot)$ are $\left\{\mathscr{F}_{t}\right\}_{t \geq 0}$-adapted, BSDE (19) can reduce to (15). This implies that $\mathrm{BSDE}$ (15) has a unique solution $(p(\cdot), \Lambda(\cdot))$ under Assumption 4. Moreover, $(p(\cdot), \widetilde{\Lambda}(\cdot))$ is the unique solution of BSDE (19), where $\widetilde{\Lambda}(\cdot)=\left(\Lambda(\cdot)^{\prime}, 0\right)^{\prime}$.

From the discussion of Section 4 in [22], we have the following lemma.

Lemma 6. If Assumption 4 holds, then

$$
\left(\begin{array}{c}
\widetilde{X}(t) \\
\widetilde{Y}(t)
\end{array}\right)=\left(\begin{array}{c}
W(t) \\
\widetilde{B}(t)
\end{array}\right)+\int_{0}^{t}\left(\begin{array}{c}
\theta(s) \\
-\frac{\widetilde{\Lambda}_{2}(s)}{p(s)}
\end{array}\right) d s
$$

is a standard Brownian motion under $\bar{P}$ where

$$
\begin{aligned}
& \frac{d \bar{P}}{d P}:=\exp \left\{-\int_{0}^{T} \theta(s)^{\prime} d W(s)+\int_{0}^{T} \frac{\widetilde{\Lambda}_{2}(s)^{\prime}}{p(s)} d \widetilde{B}(s)\right. \\
& \left.\quad-\frac{1}{2} \int_{0}^{T}\left(|\theta(s)|^{2}+\left|\frac{\widetilde{\Lambda}_{2}(s)}{p(s)}\right|^{2}\right) d s\right\} .
\end{aligned}
$$

For the existence and uniqueness of solution of BSDE (16), we have the following result.

Proposition 7. If Assumption 4 holds, then BSDE (16) has a unique solution.

Proof. The assumption guarantees that there is a unique optimal control $\widehat{\pi}(\cdot) \in \mathscr{L}_{\widehat{F}}^{2}\left(0, T ; \mathbb{R}^{m}\right)$ for $(13)$. Denote by $\widehat{X}(\cdot)$ the net wealth process associated with the optimal control $\widehat{\pi}(\cdot)$. The optimal condition (see [26]) implies that

$$
b(t)^{\prime} y(t)+\sigma(t) z_{1}(t)=0
$$

where $(y(\cdot), z(\cdot))$ is the unique solution of the following linear BSDE (called adjoint equation):

$$
\begin{aligned}
d y(t)= & -r(t) y(t) d t+z_{1}(t)^{\prime} d W(t)+z_{2}(t)^{\prime} d B(t) \\
& +z_{0}(t) d W^{0}(t), \\
y(T)= & \widehat{X}(T)-l .
\end{aligned}
$$

By using Itô's formula, we have

$$
\begin{aligned}
& d\left(\frac{1}{p(t)}\right)=\left(\frac{2 r(t)-|\theta(t)|^{2}}{p(t)}-\frac{2 \theta(t)^{\prime} \Lambda_{1}(t)}{p^{2}(t)}\right. \\
& \left.+\frac{\left|\Lambda_{2}(t)\right|^{2}}{p^{3}(t)}\right) d t-\frac{\Lambda_{1}(t)^{\prime}}{p^{2}(t)} d W(t)-\frac{\Lambda_{2}(t)^{\prime}}{p^{2}(t)} d B(t), \\
& d\left(\frac{y(t)}{p(t)}\right)=\left[\frac{\left(r(t)-|\theta(t)|^{2}\right) y(t)}{p(t)}\right. \\
& -\frac{2 \theta(t)^{\prime} \Lambda_{1}(t) y(t)+\Lambda_{1}(t)^{\prime} z_{1}(t)+\Lambda_{2}(t)^{\prime} z_{2}(t)}{p^{2}(t)} \\
& \left.+\frac{y(t)\left|\Lambda_{2}(t)\right|^{2}}{p^{3}(t)}\right] d t+\left(\frac{z_{1}(t)^{\prime}}{p(t)}-\frac{y(t) \Lambda_{1}(t)^{\prime}}{p^{2}(t)}\right) d W(t) \\
& +\left(\frac{z_{2}(t)^{\prime}}{p(t)}-\frac{y(t) \Lambda_{2}(t)^{\prime}}{p^{2}(t)}\right) d B(t)+\frac{z_{0}(t)}{p(t)} d W^{0}(t), \\
& d\left(\widehat{X}(t)-\frac{y(t)}{p(t)}\right)=\left[r(t)\left(\widehat{X}(t)-\frac{y(t)}{p(t)}\right)+\theta(t)^{\prime}\right. \\
& \left.\cdot\left(\sigma(t)^{\prime} \hat{\pi}(t)+\delta_{1}(t)-\frac{z_{1}(t)}{p(t)}+\frac{y(t) \Lambda_{1}(t)}{p^{2}(t)}\right)\right] d t \\
& -\left[\frac{\Lambda_{2}(t)^{\prime}}{p(t)}\left(\delta_{2}(t)-\frac{z_{2}(t)}{p(t)}+\frac{y(t) \Lambda_{2}(t)}{p^{2}(t)}\right)+u(t)\right. \\
& \left.+\theta^{\prime}(t) \delta_{1}(t)-\frac{\delta_{2}(t)^{\prime} \Lambda_{2}(t)}{p(t)}\right] d t+\left[\frac{y(t)|\theta(t)|^{2}}{p(t)}\right. \\
& \left.+\frac{\theta(t)^{\prime} z_{1}(t)}{p(t)}+\frac{y(t) \theta(t)^{\prime} \Lambda_{1}(t)+\Lambda_{1}(t)^{\prime} z_{1}(t)}{p^{2}(t)}\right] d t \\
& +\left(\sigma(t)^{\prime} \widehat{\pi}(t)+\delta_{1}(t)-\frac{z_{1}(t)}{p(t)}+\frac{y(t) \Lambda_{1}(t)}{p^{2}(t)}\right)^{\prime} d W(t) \\
& +\left(\delta_{2}(t)-\frac{z_{2}(t)}{p(t)}+\frac{y(t) \Lambda_{2}(t)}{p^{2}(t)}\right)^{\prime} d B(t)+\left(\delta_{0}(t)\right. \\
& \left.-\frac{z_{0}(t)}{p(t)}\right) d W^{0}(t) \text {. }
\end{aligned}
$$

It follows from (27) that $\theta(t) y(t)+z_{1}(t)=0$. Further,

$$
\begin{aligned}
& d\left(\widehat{X}(t)-\frac{y(t)}{p(t)}\right)=\left[r(t)\left(\widehat{X}(t)-\frac{y(t)}{p(t)}\right)+\theta(t)^{\prime}\right. \\
& \left.\cdot\left(\sigma(t)^{\prime} \widehat{\pi}(t)+\delta_{1}(t)-\frac{z_{1}(t)}{p(t)}+\frac{y(t) \Lambda_{1}(t)}{p^{2}(t)}\right)\right] d t \\
& -\left[\frac{\Lambda_{2}(t)^{\prime}}{p(t)}\left(\delta_{2}(t)-\frac{z_{2}(t)}{p(t)}+\frac{y(t) \Lambda_{2}(t)}{p^{2}(t)}\right)+u(t)\right. \\
& \left.+\theta(t)^{\prime} \delta_{1}(t)-\frac{\delta_{2}(t)^{\prime} \Lambda_{2}(t)}{p(t)}\right] d t+\left(\sigma(t)^{\prime} \hat{\pi}(t)\right. \\
& \left.+\delta_{1}(t)-\frac{z_{1}(t)}{p(t)}+\frac{y(t) \Lambda_{1}(t)}{p^{2}(t)}\right)^{\prime} d W(t)+\left(\delta_{2}(t)\right. \\
& \left.-\frac{z_{2}(t)}{p(t)}+\frac{y(t) \Lambda_{2}(t)}{p^{2}(t)}\right)^{\prime} d B(t)+\left(\delta_{0}(t)-\frac{z_{0}(t)}{p(t)}\right) d W^{0}(t) .
\end{aligned}
$$


Comparing with BSDE (16), we conclude that $(\widetilde{h}(\cdot), \widetilde{\eta}(\cdot))$ is a solution of (16), where

$$
\begin{aligned}
& \widetilde{h}(t):=\widehat{X}(t)-\frac{y(t)}{p(t)}, \\
& \widetilde{\eta}_{1}(t):=\sigma(t)^{\prime} \widehat{\pi}(t)+\delta_{1}(t)-\frac{z_{1}(t)}{p(t)}+\frac{y(t) \Lambda_{1}(t)}{p^{2}(t)}, \\
& \widetilde{\eta}_{2}(t):=\delta_{2}(t)-\frac{z_{2}(t)}{p(t)}+\frac{y(t) \Lambda_{2}(t)}{p^{2}(t)} \\
& \widetilde{\eta}_{0}(t):=\delta_{0}(t)-\frac{z_{0}(t)}{p(t)} .
\end{aligned}
$$

Now we show the uniqueness of the solution for (16). Assume that $(h(\cdot), \eta(\cdot))$ and $(\widetilde{h}(\cdot), \widetilde{\eta}(\cdot))$ are two solutions of BSDE (16). It follows from Itô's formula that

$d \Delta h(t)$

$$
\begin{aligned}
& =\left(r(t) \Delta h(t)+\theta(t)^{\prime} \Delta \eta_{1}(t)-\frac{\Lambda_{2}(t)^{\prime}}{p(t)} \Delta \eta_{2}(t)\right) d t \\
& \quad+\Delta \eta_{1}(t)^{\prime} d W(t)+\Delta \eta_{2}(t)^{\prime} d B(t) \\
& \quad+\Delta \eta_{0}(t) d W^{0}(t)
\end{aligned}
$$

$$
\Delta h(T)=0
$$

where $\Delta h(\cdot):=h(\cdot)-\tilde{h}(\cdot), \Delta \eta_{1}(\cdot):=\eta_{1}(\cdot)-\tilde{\eta}_{1}(\cdot), \Delta \eta_{2}(\cdot):=$ $\eta_{2}(\cdot)-\tilde{\eta}_{2}(\cdot)$, and $\Delta \eta_{0}(\cdot):=\eta_{0}(\cdot)-\tilde{\eta}_{0}(\cdot)$. have

By using the transformation defined by (25) to (32), we

$$
\begin{aligned}
d \Delta h(t)= & r(t) \Delta h(t) d t+\Delta \eta_{1}(t)^{\prime} d \widetilde{X}(t) \\
& +\left(\Delta \eta_{2}(t)^{\prime}, \Delta \eta_{0}(t)\right) d \widetilde{Y}(t), \\
\Delta h(T)= & 0
\end{aligned}
$$

which is a linear BSDE and has a unique solution $(0,0)$ under Assumption 4 . In consequence, we have $h(\cdot)=\widetilde{h}(\cdot)$ and $\eta(\cdot)=$ $\widetilde{\eta}(\cdot)$.

This completes the proof. [22].

The following lemma is a generalization of Lemma 3.1 in

Lemma 8. Suppose that Assumption 4 holds. Let $\bar{\pi}(\cdot) \in$ $\mathscr{L}_{\overparen{F}}^{2, l o c}\left(0, T ; \mathbb{R}^{m}\right)$ be given and fixed. If net wealth equation (8) corresponding to $\bar{\pi}(\cdot)$ has a unique solution $X(\cdot)$ such that $X(\cdot) \in \mathscr{L}_{\overparen{F}}^{2}(0, T ; \mathbb{R})$ and $X(T) \in \mathscr{L}_{\mathscr{F}_{T}}^{2}(\Omega ; \mathbb{R})$, then $\bar{\pi}(\cdot) \in$ $\mathscr{L}_{\overparen{F}}^{2}\left(0, T ; \mathbb{R}^{m}\right)$ is admissible.
Proof. Assume that $\bar{\pi}(\cdot) \in \mathscr{L}_{\overline{\mathscr{F}}}^{2 \text {,loc }}\left(0, T ; \mathbb{R}^{m}\right)$ is given and fixed and $\operatorname{SDE}(8)$ corresponding to $\bar{\pi}(\cdot)$ has a unique solution $X(\cdot) \in \mathscr{L}_{\overparen{F}}^{2}(0, T ; \mathbb{R})$. It follows from Itô's formula that

$$
\begin{aligned}
& X(t)^{2}=X(0)^{2}+\int_{0}^{t} 2 X(s)(r(s) X(s)+b(s) \bar{\pi}(s) \\
& -u(s)) d s+\int_{0}^{t}\left(\left|\sigma(s)^{\prime} \bar{\pi}(s)+\delta_{1}(s)\right|^{2}+\left|\delta_{2}(s)\right|^{2}\right. \\
& \left.+\left|\delta_{0}(s)\right|^{2}\right) d s+\int_{0}^{t} 2 X(s) \delta_{1}(s)^{\prime} d W(s) \\
& +\int_{0}^{t} 2 X(s) \delta_{2}(s)^{\prime} d B(s)+\int_{0}^{t} 2 X(s) \\
& \cdot \delta_{0}(s) d W^{0}(s)+\int_{0}^{t} 2 X(s) \\
& \cdot\left(\sigma(s)^{\prime} \bar{\pi}(s)\right)^{\prime} d W(s) .
\end{aligned}
$$

Under Assumption 4 , we have $2 X(\cdot) \delta_{1}(\cdot) \in \mathscr{L}_{\overparen{F}}^{2}\left(0, T ; \mathbb{R}^{m}\right)$, $2 X(\cdot) \delta_{2}(\cdot) \in \mathscr{L}_{\overparen{F}}^{2}\left(0, T ; \mathbb{R}^{d}\right)$, and $2 X(\cdot) \delta_{0}(\cdot) \in \mathscr{L}_{\overparen{F}}^{2}(0, T ; \mathbb{R})$, which imply that

$$
\begin{aligned}
& \int_{0}^{t} 2 X(s) \delta_{1}(s)^{\prime} d W(s)+\int_{0}^{t} 2 X(s) \delta_{2}(s)^{\prime} d B(s) \\
& +\int_{0}^{t} 2 X(s) \delta_{0}(s) d W^{0}(s)
\end{aligned}
$$

is a martingale and so

$$
\begin{aligned}
& E\left[\int_{0}^{t} 2 X(s) \delta_{1}(s)^{\prime} d W(s)+\int_{0}^{t} 2 X(s) \delta_{2}(s)^{\prime} d B(s)\right. \\
& \left.\quad+\int_{0}^{t} 2 X(s) \delta_{0}(s) d W^{0}(s)\right]=0 .
\end{aligned}
$$

Because $X(\cdot)$ is continuous (and bounded on $[0, T]$, a.s. $)$, we have $2 X(\cdot) \sigma(\cdot)^{\prime} \bar{\pi}(\cdot) \in \mathscr{L}_{\overparen{F}}^{2, \text { loc }}\left(0, T ; \mathbb{R}^{m}\right)$ and $\int_{0}^{t} 2 X(s)\left(\sigma(s)^{\prime} \bar{\pi}(s)\right)^{\prime} d W(s)$ is a local martingale. Therefore, there exists a localizing sequence $\left\{\tau_{i}\right\}$ for the local martingale such that

$$
\int_{0}^{t \wedge \tau_{i}} 2 X(s)\left(\sigma(s)^{\prime} \bar{\pi}(s)\right)^{\prime} d W(s)
$$

is a martingale.

Putting $t=T \wedge \tau_{i}$ and taking expectations on both sides of (34), we have

$$
\begin{aligned}
E X & \left(T \wedge \tau_{i}\right)^{2}=X(0)^{2}+E \int_{0}^{T \wedge \tau_{i}} 2 X(s)(r(s) X(s) \\
& +b(s) \bar{\pi}(s)-u(s)) d s \\
& +E \int_{0}^{T \wedge \tau_{i}}\left(\left|\sigma(s)^{\prime} \bar{\pi}(s)+\delta_{1}(s)\right|^{2}+\left|\delta_{2}(s)\right|^{2}\right. \\
& \left.+\left|\delta_{0}(s)\right|^{2}\right) d s .
\end{aligned}
$$


Then it can be rewritten as

$$
\begin{aligned}
& X(0)^{2}+E \int_{0}^{T \wedge \tau_{i}} \bar{\pi}(s)^{\prime} \sigma(s) \sigma(s)^{\prime} \bar{\pi}(s) d s \\
& +E \int_{0}^{T \wedge \tau_{i}}\left(\left|\delta_{1}(s)\right|^{2}+\left|\delta_{2}(s)\right|^{2}+\left|\delta_{0}(s)\right|^{2}\right) d s \\
& =E X\left(T \wedge \tau_{i}\right)^{2}-E \int_{0}^{T \wedge \tau_{i}} 2 r(s) X(s)^{2} d s \\
& +E \int_{0}^{T \wedge \tau_{i}} 2 X(s) u(s) d s \\
& -E \int_{0}^{T \wedge \tau_{i}} 2 \delta_{1}(s)^{\prime} \sigma(s)^{\prime} \bar{\pi}(s) d s \\
& \quad-E \int_{0}^{T \wedge \tau_{i}} 2 X(s) b(s) \bar{\pi}(s) d s .
\end{aligned}
$$

Since

$$
\begin{aligned}
& -2 X(s) b(s) \bar{\pi}(s)=-2\left(\sqrt{\frac{4}{\epsilon}} X(s) b(s)\right)\left(\sqrt{\frac{\epsilon}{4}} \bar{\pi}(s)\right) \\
& \leq \frac{4}{\epsilon} X(s)^{2}|b(s)|^{2}+\frac{\epsilon}{4}|\bar{\pi}(s)|^{2} \\
& -2 \delta_{1}(s)^{\prime} \sigma(s)^{\prime} \bar{\pi}(s) \\
& =-2\left(\sqrt{\frac{4}{\epsilon}} \delta_{1}(s)^{\prime} \sigma(s)^{\prime}\right)\left(\sqrt{\frac{\epsilon}{4}} \bar{\pi}(s)\right) \\
& \leq \frac{4}{\epsilon}\left|\sigma(s) \delta_{1}(s)\right|^{2}+\frac{\epsilon}{4}|\bar{\pi}(s)|^{2}
\end{aligned}
$$

we have

$$
\begin{aligned}
& X(0)^{2}+E \int_{0}^{T \wedge \tau_{i}} \bar{\pi}(s)^{\prime} \sigma(s) \sigma(s)^{\prime} \bar{\pi}(s) d s \\
& +E \int_{0}^{T \wedge \tau_{i}}\left(\left|\delta_{1}(s)\right|^{2}+\left|\delta_{2}(s)\right|^{2}+\left|\delta_{0}(s)\right|^{2}\right) d s \\
& \leq E X\left(T \wedge \tau_{i}\right)^{2}-E \int_{0}^{T \wedge \tau_{i}} 2 r(s) X(s)^{2} d s \\
& +E \int_{0}^{T \wedge \tau_{i}} 2 X(s) u(s) d s \\
& +E \int_{0}^{T \wedge \tau_{i}} \frac{4}{\epsilon}\left|\sigma(s) \delta_{1}(s)\right|^{2}+\frac{\epsilon}{4}|\bar{\pi}(s)|^{2} d s \\
& \quad+E \int_{0}^{T \wedge \tau_{i}} \frac{4}{\epsilon} X(s)^{2}|b(s)|^{2}+\frac{\epsilon}{4}|\bar{\pi}(s)|^{2} d s
\end{aligned}
$$

Rewriting the inequality above, we have

$$
\begin{aligned}
X(0)^{2} & +E \int_{0}^{T \wedge \tau_{i}}\left(\left|\delta_{1}(s)\right|^{2}+\left|\delta_{2}(s)\right|^{2}+\left|\delta_{0}(s)\right|^{2}\right) d s \\
& +E \int_{0}^{T \wedge \tau_{i}} \bar{\pi}(s)^{\prime} \sigma(s) \sigma(s)^{\prime} \bar{\pi}(s) d s \\
& -\frac{\epsilon}{2} E \int_{0}^{T \wedge \tau_{i}}|\bar{\pi}(s)|^{2} d s \\
\leq & E X\left(T \wedge \tau_{i}\right)^{2} \\
& +E \int_{0}^{T \wedge \tau_{i}} X(s)^{2}\left(-2 r(s)+\frac{4}{\epsilon}|b(s)|^{2}\right) d s \\
& +E \int_{0}^{T \wedge \tau_{i}} 2 X(s) u(s) d s \\
& +\frac{4}{\epsilon} E \int_{0}^{T \wedge \tau_{i}}\left|\sigma(s) \delta_{1}(s)\right|^{2} d s .
\end{aligned}
$$

From Fatou's lemma, we obtain

$$
\begin{aligned}
& X(0)^{2}+E \int_{0}^{T}\left(\left|\delta_{1}(s)\right|^{2}+\left|\delta_{2}(s)\right|^{2}+\left|\delta_{0}(s)\right|^{2}\right) d s \\
& +E \int_{0}^{T} \bar{\pi}(s)^{\prime} \sigma(s) \sigma(s)^{\prime} \bar{\pi}(s) d s \\
& \quad-\frac{\epsilon}{2} E \int_{0}^{T}|\bar{\pi}(s)|^{2} d s \leq E X(T)^{2} \\
& +E \int_{0}^{T} X(s)^{2}\left(-2 r(s)+\frac{4}{\epsilon}|b(s)|^{2}\right) d s \\
& +E \int_{0}^{T} 2 X(s) u(s) d s+\frac{4}{\epsilon} E \int_{0}^{T}\left|\sigma(s) \delta_{1}(s)\right|^{2} d s \\
& <+\infty,
\end{aligned}
$$

where the last inequality comes from Assumption 4 and $X(\cdot) \in \mathscr{L}_{\overparen{F}}^{2}(0, T ; \mathbb{R})$.

Since $\sigma(t) \sigma(t)^{\prime} \geq \epsilon I_{m}$, we have

$$
\begin{aligned}
& \frac{\epsilon}{2} E \int_{0}^{T}|\bar{\pi}(s)|^{2} d s \\
& \leq X(0)^{2} \\
& \quad+E \int_{0}^{T}\left(\left|\delta_{1}(s)\right|^{2}+\left|\delta_{2}(s)\right|^{2}+\left|\delta_{0}(s)\right|^{2}\right) d s \\
& \quad+E \int_{0}^{T} \bar{\pi}(s)^{\prime} \sigma(s) \sigma(s)^{\prime} \bar{\pi}(s) d s \\
& \quad-\frac{\epsilon}{2} E \int_{0}^{T}|\bar{\pi}(s)|^{2} d s<+\infty
\end{aligned}
$$

which implies that $\bar{\pi}(\cdot) \in \mathscr{L}_{\widehat{\mathscr{F}}}^{2}\left(0, T ; \mathbb{R}^{m}\right)$ is admissible.

$$
\text { This completes the proof. }
$$


The following result concerns the admissibility of (46).

Proposition 9. If Assumption 4 holds, then

$$
\begin{aligned}
& d X(t)=\left\{r(t) X(t)-\theta(t)^{\prime}\left[\delta_{1}(t)-\eta_{1}(t)\right.\right. \\
& \left.\left.+(X(t)-h(t))\left(\theta(t)+\frac{\Lambda_{1}(t)}{p(t)}\right)\right]-u(t)\right\} d t \\
& +\left[\eta_{1}(t)-(X(t)-h(t))\left(\theta(t)+\frac{\Lambda_{1}(t)}{p(t)}\right)\right]^{\prime} d W(t) \\
& +\delta_{2}(t)^{\prime} d B(t)+\delta_{0}(t) d W^{0}(t), \\
& X(0)=X_{0}
\end{aligned}
$$

(replace (8) by (46)) has a unique solution with $X(\cdot) \in$ $\mathscr{L}_{\overparen{F}}^{2}(0, T ; \mathbb{R})$ and $X(T) \in \mathscr{L}_{\widetilde{F}_{T}}^{2}(\Omega ; \mathbb{R})$. Moreover,

$$
\begin{gathered}
\bar{\pi}(t):=-\left(\sigma(t)^{-1}\right)^{\prime}\left[\delta_{1}(t)-\eta_{1}(t)\right. \\
\left.+(X(t)-h(t))\left(\theta(t)+\frac{\Lambda_{1}(t)}{p(t)}\right)\right]
\end{gathered}
$$

is admissible.

Proof. Consider the following SDE:

$$
\begin{aligned}
d Y(t)= & -r(t) Y(t) d t-Y(t) \theta(t)^{\prime} d W(t) \\
& +\left[Y(t) \alpha_{1}(t)+\alpha_{2}(t)\right]^{\prime} d B(t) \\
& +\beta_{2}(t) d W^{0}(t), \\
Y(0)= & p(0)(X(0)-h(0)),
\end{aligned}
$$

where

$$
\begin{aligned}
& \alpha_{1}(t)=\frac{\Lambda_{2}(t)}{p(t)}, \\
& \alpha_{2}(t)=p(t)\left(\delta_{2}(t)-\eta_{2}(t)\right), \\
& \beta_{2}(t)=p(t)\left(\delta_{0}(t)-\eta_{0}(t)\right) .
\end{aligned}
$$

It can be shown that

$$
\begin{aligned}
& Y(t)=\Phi(t)[p(0)(X(0)-h(0)) \\
& \left.-\int_{0}^{t} \alpha_{1}(s)^{\prime} \alpha_{2}(s) \Phi^{-1}(s) d s\right]+\Phi(t) \\
& \cdot\left(\int_{0}^{t} \alpha_{2}(s)^{\prime} \Phi^{-1}(s) d B(s)\right. \\
& \left.+\int_{0}^{t} \beta_{2}(s) \Phi^{-1}(s) d W^{0}(s)\right)
\end{aligned}
$$

is the unique solution of (47), where

$$
\begin{gathered}
\Phi(t)=\exp \left\{-\frac{1}{2} \int_{0}^{t}\left(|\theta(s)|^{2}+\left|\alpha_{1}(s)\right|^{2}+2 r(s)\right) d s\right. \\
\left.-\int_{0}^{t} \theta(s)^{\prime} d W(s)+\int_{0}^{t} \alpha_{1}(s)^{\prime} d B(s)\right\} .
\end{gathered}
$$

By using Itô's formula, we have that

$$
X(t)=h(t)+\frac{Y(t)}{p(t)}
$$

is the unique solution of SDE (45).

It follows from Itô's formula that

$$
\begin{aligned}
& d(X(t)-h(t))=[r(t)(X(t)-h(t)) \\
& -(X(t)-h(t)) \theta(t)^{\prime}\left(\theta(t)+\frac{\Lambda_{1}(t)}{p(t)}\right) \\
& \left.+\frac{\Lambda_{2}(t)^{\prime}}{p(t)}\left(\eta_{2}(t)-\delta_{2}(t)\right)\right] d t-(X(t)-h(t)) \\
& \cdot\left(\theta(t)+\frac{\Lambda_{1}(t)}{p(t)}\right)^{\prime} d W(t) \\
& +\left(\delta_{2}(t)-\eta_{2}(t)\right)^{\prime} d B(t)+\left(\delta_{0}(t)-\eta_{0}(t)\right) d W^{0}(t), \\
& d(X(t)-h(t))^{2} \\
& =\left[(X(t)-h(t))^{2}\left(2 r(t)-|\theta(t)|^{2}+\left|\frac{\Lambda_{1}(t)}{p(t)}\right|^{2}\right)\right. \\
& \left.+2(X(t)-h(t))\left(\eta_{2}(t)-\delta_{2}(t)\right)^{\prime} \frac{\Lambda_{2}(t)}{p(t)}\right] d t \\
& +\left(\left|\delta_{2}(t)-\eta_{2}(t)\right|^{2}+\left|\delta_{0}(t)-\eta_{0}(t)\right|^{2}\right) d t \\
& -2(X(t)-h(t))^{2}\left(\theta(t)+\frac{\Lambda_{1}(t)}{p(t)}\right)^{\prime} d W(t) \\
& +2(X(t)-h(t))\left(\delta_{2}(t)-\eta_{2}(t)\right)^{\prime} d B(t)+2(X(t) \\
& -h(t))\left(\delta_{0}(t)-\eta_{0}(t)\right) d W^{0}(t), \\
& d p(t)(X(t)-h(t))^{2}=p(t)\left(\left|\delta_{2}(t)-\eta_{2}(t)\right|^{2}\right. \\
& \left.+\left|\delta_{0}(t)-\eta_{0}(t)\right|^{2}\right) d t-p(t)(X(t)-h(t))^{2}(2 \theta(t) \\
& \left.+\frac{\Lambda_{1}(t)}{p(t)}\right)^{\prime} d W(t) \\
& +\left[2 p(t)(X(t)-h(t))\left(\delta_{2}(t)-\eta_{2}(t)\right)^{\prime}\right. \\
& \left.+(X(t)-h(t))^{2} \Lambda_{2}(t)^{\prime}\right] d B(t)+2 p(t)(X(t) \\
& -h(t))\left(\delta_{0}(t)-\eta_{0}(t)\right) d W^{0}(t) .
\end{aligned}
$$


Then, we have

$$
\begin{aligned}
& p(t)(X(t)-h(t))^{2}=p(0)(X(0)-h(0))^{2} \\
& +\int_{0}^{t} p(s)\left(\left|\delta_{2}(s)-\eta_{2}(s)\right|^{2}+\left|\delta_{0}(s)-\eta_{0}(s)\right|^{2}\right) d s \\
& -\int_{0}^{t} p(s)(X(s)-h(s))^{2}(2 \theta(s) \\
& \left.+\frac{\Lambda_{1}(s)}{p(s)}\right)^{\prime} d W(s) \\
& +\int_{0}^{t}\left[2 p(s)(X(s)-h(s))\left(\delta_{2}(s)-\eta_{2}(s)\right)^{\prime}\right. \\
& \left.+(X(s)-h(s))^{2} \Lambda_{2}(s)^{\prime}\right] d B(s)+\int_{0}^{t} 2 p(s) \\
& \cdot(X(s)-h(s))\left(\delta_{0}(s)-\eta_{0}(s)\right) d W^{0}(s)
\end{aligned}
$$

Taking $X(t)=h(t)+Y(t) / p(t)$ into account, we conclude that

$$
\begin{aligned}
& -\int_{0}^{t} p(s)(X(s)-h(s))^{2}\left(2 \theta(s)+\frac{\Lambda_{1}(s)}{p(s)}\right)^{\prime} d W(s) \\
& +\int_{0}^{t} 2 p(s)(X(s)-h(s))\left(\delta_{2}(s)-\eta_{2}(s)\right)^{\prime} d B(s) \\
& +\int_{0}^{t}(X(s)-h(s))^{2} \Lambda_{2}(s)^{\prime} d B(s) \\
& +\int_{0}^{t} 2 p(s)(X(s)-h(s))\left(\delta_{0}(s)-\eta_{0}(s)\right) d W^{0}(s) \\
& =-\int_{0}^{t} \frac{Y(s)^{2}}{p(s)}\left(2 \theta(s)+\frac{\Lambda_{1}(s)}{p(s)}\right)^{\prime} d W(s) \\
& +\int_{0}^{t} 2 Y(s)\left(\delta_{2}(s)-\eta_{2}(s)\right)^{\prime} d B(s) \\
& +\int_{0}^{t} \frac{Y(s)^{2}}{p(s)^{2}} \Lambda_{2}(s)^{\prime} d B(s) \\
& +\int_{0}^{t} 2 Y(s)\left(\delta_{0}(s)-\eta_{0}(s)\right) d W^{0}(s)
\end{aligned}
$$

is a local martingale under Assumption 4. Let $\left\{\tau_{i}\right\}$ be a localizing sequence for the local martingale above. Then, for any $t \in[0, T]$,

$$
\begin{gathered}
E\left[p\left(t \wedge \tau_{i}\right)\left(X\left(t \wedge \tau_{i}\right)-h\left(t \wedge \tau_{i}\right)\right)^{2}\right]=p(0)(X(0) \\
-h(0))^{2}+E \int_{0}^{t \wedge \tau_{i}} p(s) \\
\cdot\left(\left|\delta_{2}(s)-\eta_{2}(s)\right|^{2}+\left|\delta_{0}(s)-\eta_{0}(s)\right|^{2}\right) d s .
\end{gathered}
$$

It follows from Fatou's lemma and Assumption 4 that

$$
\begin{gathered}
E\left[p(t)(X(t)-h(t))^{2}\right] \leq p(0)(X(0)-h(0))^{2} \\
+E \int_{0}^{t} p(s) \\
\cdot\left(\left|\delta_{2}(s)-\eta_{2}(s)\right|^{2}+\left|\delta_{0}(s)-\eta_{0}(s)\right|^{2}\right) d s \\
\leq p(0)(X(0)-h(0))^{2}+E \int_{0}^{T} p(s) \\
\cdot\left(\left|\delta_{2}(s)-\eta_{2}(s)\right|^{2}+\left|\delta_{0}(s)-\eta_{0}(s)\right|^{2}\right) d s .
\end{gathered}
$$

Since $p(\cdot)>0$ and $1 / p(\cdot) \in \mathscr{L}_{\mathscr{F}}^{\infty}(\Omega ; C(0, T ; \mathbb{R}))$, there exists a constant $\varepsilon>0$ such that, for any $t \in[0, T], p(t) \geq \varepsilon$. Thus, we have

$$
\varepsilon E\left[(X(t)-h(t))^{2}\right] \leq E\left[p(t)(X(t)-h(t))^{2}\right] \leq H,
$$

where $H:=p(0)(X(0)-h(0))^{2}+E \int_{0}^{T} p(s)\left(\left|\delta_{2}(s)-\eta_{2}(s)\right|^{2}+\right.$ $\left.\left|\delta_{0}(s)-\eta_{0}(s)\right|^{2}\right) d s<+\infty$.

Further, we have

$$
\begin{aligned}
& E\left[(X(T)-h(T))^{2}\right] \leq \frac{1}{\varepsilon} E\left[p(T)(X(T)-h(T))^{2}\right] \\
& \quad \leq \frac{H}{\varepsilon}<+\infty, \\
& E\left[\int_{0}^{T}(X(t)-h(t))^{2} d t\right]=\int_{0}^{T} E(X(t)-h(t))^{2} d t \\
& \quad \leq \frac{H}{\varepsilon} T<+\infty
\end{aligned}
$$

which means that $(X(T)-h(T)) \in \mathscr{L}_{\widetilde{F}_{T}}^{2}(\Omega ; \mathbb{R})$ and $(X(\cdot)-$ $h(\cdot)) \in \mathscr{L}_{\overparen{F}}^{2}(0, T ; \mathbb{R})$.

Because $h(T) \in \mathscr{L}_{\widetilde{F}_{T}}^{2}(\Omega ; \mathbb{R})$ and $h(\cdot) \in \mathscr{L}_{\overparen{\mathscr{F}}}^{2}(0, T ; \mathbb{R})$, we have

$$
\begin{aligned}
X(\cdot) & =(X(\cdot)-h(\cdot))+h(\cdot) \in \mathscr{L}_{\overparen{F}}^{2}(0, T ; \mathbb{R}), \\
X(T) & =(X(T)-h(T))+h(T) \in \mathscr{L}_{\widetilde{F}_{T}}^{2}(\Omega ; \mathbb{R}) .
\end{aligned}
$$

Since $\Lambda_{1}(\cdot) \in \mathscr{L}_{\mathscr{F}}^{2}\left(0, T ; \mathbb{R}^{m}\right)$ and $h(\cdot) \in \mathscr{L}_{\overparen{F}}^{2}(\Omega ; C(0, T ;$ $\mathbb{R})$ ), it follows from $(46)$ that $\bar{\pi}(\cdot) \in \mathscr{L}_{\widetilde{F}}^{2, \text { loc }}\left(0, T ; \mathbb{R}^{m}\right)$. Further, we conclude from Lemma 8 that $\bar{\pi}(\cdot) \in \mathscr{L}_{\overparen{\mathscr{F}}}^{2}\left(0, T ; \mathbb{R}^{m}\right)$ and $\bar{\pi}(\cdot)$ is admissible.

This completes the proof.

Next, we formulate the optimal control policy and the cost for the unconstrained control problem (13).

Theorem 10. Let $\bar{\pi}(\cdot)$ be given by (46). If Assumption 4 holds, then $\bar{\pi}(\cdot)$ is the unique optimal control policy for problem (13) and

$$
\begin{aligned}
J^{*} & =p(0)(X(0)-h(0))^{2}+E \int_{0}^{T} p(t) \\
& \cdot\left(\left|\delta_{2}(t)-\eta_{2}(t)\right|^{2}+\left|\delta_{0}(t)-\eta_{0}(t)\right|^{2}\right) d t
\end{aligned}
$$

is the optimal cost. 
Proof. From Itô's formula, (16) and (8) give

$$
\begin{aligned}
& d(X(t)-h(t))=[r(t)(X(t)-h(t))+b(t) \pi(t) \\
& \left.+\theta(t)^{\prime}\left(\delta_{1}(t)-\eta_{1}(t)\right)+\frac{\Lambda_{2}(t)^{\prime}}{p(t)}\left(\eta_{2}(t)-\delta_{2}(t)\right)\right] d t \\
& +\left(\sigma(t)^{\prime} \pi(t)+\delta_{1}(t)-\eta_{1}(t)\right)^{\prime} d W(t)+\left(\delta_{2}(t)\right. \\
& \left.-\eta_{2}(t)\right)^{\prime} d B(t)+\left(\delta^{0}(t)-\eta_{0}(t)\right) d W^{0}(t), \\
& d(X(t)-h(t))^{2}=2(X(t)-h(t)) \\
& \cdot[r(t)(X(t)-h(t)) \\
& +b(t) \pi(t)+\theta(t)^{\prime}\left(\delta_{1}(t)-\eta_{1}(t)\right) \\
& \left.+\frac{\Lambda_{2}(t)^{\prime}}{p(t)}\left(\eta_{2}(t)-\delta_{2}(t)\right)\right] d t+2(X(t)-h(t)) \\
& \cdot\left(\sigma(t)^{\prime} \pi(t)+\delta_{1}(t)\right. \\
& \left.-\eta_{1}(t)\right)^{\prime} d W(t)+2(X(t)-h(t))\left(\delta_{2}(t)-\eta_{2}(t)\right)^{\prime} d B(t) \\
& +2(X(t)-h(t))\left(\delta_{0}(t)-\eta_{0}(t)\right) d W^{0}(t) \\
& +\left(\left|\sigma(t)^{\prime} \pi(t)+\delta_{1}(t)-\eta_{1}(t)\right|^{2}+\left|\delta_{2}(t)-\eta_{2}(t)\right|^{2}\right. \\
& \left.+\left(\delta_{0}(t)-\eta_{0}(t)\right)^{2}\right) d t .
\end{aligned}
$$

By using Itô's formula again, we have

$$
\begin{aligned}
d p & (t)(X(t)-h(t))^{2}=p(t)\left\{\pi(t)^{\prime} \sigma(t) \sigma(t)^{\prime} \pi(t)\right. \\
+ & 2 \pi(t)^{\prime}\left[\sigma(t)\left(\delta_{1}(t)-\eta_{1}(t)\right)\right. \\
+ & \left.\left.(X(t)-h(t))\left(b(t)^{\prime}+\frac{\sigma(t) \Lambda_{1}(t)}{p(t)}\right)\right]\right\} d t \\
+ & p(t)\left[\left|\delta_{0}(t)-\eta_{0}(t)\right|^{2}+\left|\delta_{2}(t)-\eta_{2}(t)\right|^{2}\right. \\
+ & \mid \delta_{1}(t)-\eta_{1}(t) \\
+ & \left.\left.(X(t)-h(t))\left(\theta(t)+\frac{\Lambda_{1}(t)}{p(t)}\right)\right|^{2}\right] d t+(X(t) \\
& +h(t))\left[(X(t)-h(t)) \Lambda_{1}(t)+2 p(t)\left(\sigma(t)^{\prime} \pi(t)\right.\right. \\
+ & \left.\left.\delta_{1}(t)-\eta_{1}(t)\right)\right]^{\prime} d W(t)+(X(t)-h(t))[(X(t) \\
- & \left.h(t)) \Lambda_{2}(t)+2 p(t)\left(\delta_{2}(t)-\eta_{2}(t)\right)\right]^{\prime} d B(t) \\
+ & 2 p(t)(X(t)-h(t))\left(\delta_{0}(t)-\eta_{0}(t)\right) d W^{0}(t)
\end{aligned}
$$

or

$$
\begin{aligned}
d p & (t)(X(t)-h(t))^{2}=p(t) \\
\cdot & {\left[(\pi(t)-\bar{\pi}(t))^{\prime} \sigma(t) \sigma(t)^{\prime}(\pi(t)-\bar{\pi}(t))\right] d t } \\
+ & p(t)\left[\left|\delta_{2}(t)-\eta_{2}(t)\right|^{2}+\left|\delta_{0}(t)-\eta_{0}(t)\right|^{2}\right] d t \\
+ & (X(t)-h(t))\left[(X(t)-h(t)) \Lambda_{1}(t)\right. \\
+ & \left.2 p(t)\left(\sigma(t)^{\prime} \pi(t)+\delta_{1}(t)-\eta_{1}(t)\right)\right]^{\prime} d W(t) \\
+ & (X(t)-h(t))\left[(X(t)-h(t)) \Lambda_{2}(t)\right. \\
+ & \left.2 p(t)\left(\delta_{2}(t)-\eta_{2}(t)\right)\right]^{\prime} d B(t)+2 p(t)(X(t) \\
& -h(t))\left(\delta_{0}(t)-\eta_{0}(t)\right) d W^{0}(t),
\end{aligned}
$$

where $\bar{\pi}(t)$ is given by (46).

Then, by integrating from $[0, T]$ and taking expectations, we have

$$
\begin{aligned}
& E p(T)(X(T)-h(T))^{2}=p(0)(X(0)-h(0))^{2} \\
& +E \int_{0}^{T} p(t)\left[(\pi(t)-\bar{\pi}(t))^{\prime} \sigma(t) \sigma(t)^{\prime}\right. \\
& \cdot(\pi(t)-\bar{\pi}(t))] d t+E \int_{0}^{T} p(t)\left(\left|\delta_{2}(t)-\eta_{2}(t)\right|^{2}\right. \\
& \left.+\left|\delta_{0}(t)-\eta_{0}(t)\right|^{2}\right) d t+E \int_{0}^{T}(X(t)-h(t)) \\
& \cdot\left[(X(t)-h(t)) \Lambda_{1}(t)+2 p(t)\right. \\
& \left.\cdot\left(\sigma(t)^{\prime} \pi(t)+\delta_{1}(t)-\eta_{1}(t)\right)\right]^{\prime} d W(t) \\
& +E \int_{0}^{T}(X(t)-h(t))\left[(X(t)-h(t)) \Lambda_{2}(t)\right. \\
& \left.+2 p(t)\left(\delta_{2}(t)-\eta_{2}(t)\right)\right]^{\prime} d B(t) \\
& +E \int_{0}^{T} 2 p(t)(X(t)-h(t))\left(\delta_{0}(t)\right. \\
& \left.-\eta_{0}(t)\right) d W^{0}(t)=p(0)(X(0)-h(0))^{2} \\
& +E \int_{0}^{T} p(t)\left[(\pi(t)-\bar{\pi}(t))^{\prime} \sigma(t) \sigma(t)^{\prime}\right. \\
& \cdot(\pi(t)-\bar{\pi}(t))] d t+E \int_{0}^{T} p(t)\left(\left|\delta_{2}(t)-\eta_{2}(t)\right|^{2}\right. \\
& \left.+\left|\delta_{0}(t)-\eta_{0}(t)\right|^{2}\right) d t
\end{aligned}
$$


Due to the fact that $p(t)>0$ and $\sigma(t) \sigma(t)^{\prime}>\epsilon I_{m}$, we have

$$
\begin{gathered}
E[X(T)-l]^{2}=E P(T)(X(T)-h(T))^{2} \geq p(0) \\
\cdot(X(0)-h(0))^{2}+E \int_{0}^{T} p(t) \\
\cdot\left(\left|\delta_{2}(t)-\eta_{2}(t)\right|^{2}+\left|\delta_{0}(t)-\eta_{0}(t)\right|^{2}\right) d t,
\end{gathered}
$$

where the equality holds only when $\pi(\cdot)=\bar{\pi}(\cdot)$. This completes the proof.

Remark 11. If there is no liability, that is, $u(\cdot)=v(\cdot)=0$ and $\mathscr{F}_{t}=\widehat{\mathscr{F}}_{t}$, then $\delta(\cdot)=0$, and (16) boils down to

$$
\begin{aligned}
d h(t)= & \left(r(t) h(t)+\theta(t)^{\prime} \eta_{1}(t)-\frac{\Lambda_{2}(t)^{\prime}}{p(t)} \eta_{2}(t)\right) d t \\
& +\eta_{1}(t)^{\prime} d W(t)+\eta_{2}(t)^{\prime} d B(t) \\
& +\eta_{0}(t) d W^{0}(t), \\
h(T)= & l,
\end{aligned}
$$

and the unique optimal control policy for problem (13) is

$$
\begin{aligned}
\bar{\pi}(t) & =-\left(\sigma(t)^{-1}\right)^{\prime} \\
\cdot & {\left[-\eta_{1}(t)+(X(t)-h(t))\left(\theta(t)+\frac{\Lambda_{1}(t)}{p(t)}\right)\right], }
\end{aligned}
$$

which is the same as (12) in Lim [22].

\section{The Mean-Variance Asset-Liability Management Problem}

An admissible portfolio $\pi$ is said to be a feasible portfolio for (12) if it satisfies the constraint in (12). Then, problem (12) is said to be feasible if it has a feasible portfolio. Following the methodology of Lim [22], we get a necessary and sufficient condition for feasibility of problem (12) as follows.

Proposition 12. Let $(\Psi(\cdot), \xi(\cdot))$ be a unique solution of the following BSDE:

$$
\begin{aligned}
d \Psi(t)= & -r(t) \Psi(t) d t+\xi_{1}(t)^{\prime} d W(t) \\
& +\xi_{2}(t)^{\prime} d B(t) \\
\Psi(T)= & 1 .
\end{aligned}
$$

If Assumption 4 holds, then mean-variance problem (12) is feasible for any $c \in \mathbb{R}$ if and only if

$$
E \int_{0}^{T}\left|\Psi(t) b(t)^{\prime}+\sigma(t) \xi_{1}(t)\right|^{2} d t>0
$$

Proof. Let $\pi(\cdot)$ be admissible and $\tilde{\pi}(\cdot)=\lambda \pi(\cdot)$ for some $\lambda \in \mathbb{R}$. Assume that $\widetilde{X}(\cdot)$ is the solution of (8) corresponding to $\widetilde{\pi}(\cdot)$. It follows from Itô's formula that $\widetilde{X}(t)=Z_{1}(t)+\lambda Z_{2}(t)$, where

$$
\begin{aligned}
d Z_{1}(t)= & \left(r(t) Z_{1}(t)-u(t)\right) d t+\delta_{1}(t)^{\prime} d W(t) \\
& +\delta_{2}(t)^{\prime} d B(t)+\delta^{0}(t) d W^{0}(t), \\
Z_{1}(0)= & X_{0}, \\
d Z_{2}(t)= & \left(r(t) Z_{2}(t)+b(t) \pi(t)\right) d t \\
& +\pi(t)^{\prime} \sigma(t) d W(t), \\
Z_{2}(0)= & 0 .
\end{aligned}
$$

Then we have $E \widetilde{X}(T)=E Z_{1}(T)+\lambda E Z_{2}(T)$, where

$$
E Z_{2}(T)=E \int_{0}^{T}\left(\Psi(t) b(t)^{\prime}+\sigma(t) \xi_{1}(t)\right)^{\prime} \pi(t) d t
$$

which has been shown in Yong and Zhou [27] (see pp. 353 of [27]). If (69) holds, then we can choose $\pi(t)=\Psi(t) b(t)^{\prime}+$ $\sigma(t) \xi_{1}(t)$ such that

$$
E Z_{2}(T)=E \int_{0}^{T}\left|\Psi(t) b(t)^{\prime}+\sigma(t) \xi_{1}(t)\right|^{2} d t>0
$$

Hence, for any $c \in \mathbb{R}, \lambda_{c}=\left(E Z_{2}(T)\right)^{-1}\left(c-E Z_{1}(T)\right)$ is well defined and

$$
E \widetilde{X}(T)=E Z_{1}(T)+\lambda_{c} E Z_{2}(T)=c .
$$

This implies that (12) is feasible for any $c \in \mathbb{R}$.

Conversely, if (12) is feasible for any $c \in \mathbb{R}$, then, for any $c \in \mathbb{R}$, there exists an admissible portfolio $\pi(\cdot)$ such that $E X(T)=E Z_{1}(T)+E Z_{2}(T)=c$. Since $E Z_{1}(T)$ is independent of $\pi(\cdot)$, we conclude that $E Z_{2}(T) \neq 0$ for some $\pi(\cdot)$. From (71), we know that (69) is true.

This completes the proof.

Remark 13. The necessary and sufficient condition (69) is the same as that in [22] in which Lim studied the meanvariance portfolio problem without liability. This implies that the liability does not affect the feasibility of mean-variance problem.

Remark 14. As claimed in [22], necessary and sufficient condition (69) is very mild. 
In the case of mean-variance asset-liability management problem, we can replace the unique solution $(h(\cdot), \eta(\cdot))$ of BSDE (16) by

$$
\begin{aligned}
& h(t)=h(T) g_{1}(t)+g_{2}(t), \\
& \eta_{1}(t)=h(T) q_{1}(t)+\bar{q}_{1}(t), \\
& \eta_{2}(t)=h(T) q_{2}(t)+\bar{q}_{2}(t), \\
& \eta_{0}(t)=\bar{q}_{0}(t),
\end{aligned}
$$

where $\left(g_{1}(\cdot), q(\cdot)\right)$ and $\left(g_{2}(\cdot), \bar{q}(\cdot)\right)$ are the unique solutions of the following BSDEs:

$$
\begin{aligned}
& d g_{1}(t)=\left(r(t) g_{1}(t)+\theta(t)^{\prime} q_{1}(t)-\frac{\Lambda_{2}(t)^{\prime}}{p(t)} q_{2}(t)\right) d t \\
& \quad+q_{1}(t)^{\prime} d W(t)+q_{2}(t)^{\prime} d B(t), \\
& g_{1}(T)=1, \\
& d g_{2}(t)=\left(r(t) g_{2}(t)+\theta(t)^{\prime} \bar{q}_{1}(t)-\frac{\Lambda_{2}(t)^{\prime}}{p(t)} \bar{q}_{2}(t)\right. \\
& \left.\quad-u(t)-\theta(t)^{\prime} \delta_{1}(t)+\delta_{2}(t)^{\prime} \frac{\Lambda_{2}(t)}{p(t)}\right) d t+\bar{q}_{1}(t)^{\prime} d W(t) \\
& \quad+\bar{q}_{2}(t)^{\prime} d B(t)+\bar{q}_{0}(t) d W^{0}(t), \\
& g_{2}(T)=0,
\end{aligned}
$$

respectively.

By employing the results in Section 3 and Lagrange multiplier technique (or duality theory), we give our main result as follows.

Theorem 15. If Assumption 4 holds and (69) is satisfied, then mean-variance asset-liability management problem (12) is feasible for every $c \in \mathbb{R}$, and the inequality

$$
1-M-p(0) g_{1}^{2}(0)>0
$$

holds and the following constants,

$$
\begin{aligned}
M & :=E \int_{0}^{T} p(t)\left|q_{2}(t)\right|^{2} d t, \\
k_{1} & :=E \int_{0}^{T} p(t) q_{2}(t)^{\prime}\left(\delta_{2}(t)-\bar{q}_{2}(t)\right) d t, \\
k & :=\frac{-c+p(0) g_{1}(0)\left(X_{0}-g_{2}(0)\right)+k_{1}}{1-M-p(0) g_{1}^{2}(0)}, \\
D & :=E \int_{0}^{T} p(t) \\
& \cdot\left[\left|\delta_{2}(t)-\bar{q}_{2}(t)\right|^{2}+\left(\delta_{0}(t)-\bar{q}_{0}(t)\right)^{2}\right] d t,
\end{aligned}
$$

are well defined. The efficient frontier of problem (12) is given by

$$
\begin{aligned}
& \operatorname{Var} X^{*}(T)=\frac{M+p(0) g_{1}^{2}(0)}{1-M-p(0) g_{1}^{2}(0)}[c \\
& \left.-\frac{k_{1}+p(0) g_{1}(0)\left(X_{0}-g_{2}(0)\right)}{M+p(0) g_{1}^{2}(0)}\right]^{2}+D \\
& +p(0)\left(X_{0}-g_{2}(0)\right)^{2} \\
& -\frac{\left[k_{1}+p(0) g_{1}(0)\left(X_{0}-g_{2}(0)\right)\right]^{2}}{M+p(0) g_{1}^{2}(0)},
\end{aligned}
$$

where $c=E X^{*}(T)$ and the optimal portfolio associated with the expected net terminal wealth $c$ is given as follows:

$$
\begin{gathered}
\pi^{*}(t)=-\left(\sigma(t)^{-1}\right)^{\prime}\left[\delta_{1}(t)-\bar{q}_{1}(t)+k q_{1}(t)\right. \\
\left.+\left(X(t)-g_{2}(t)+k g_{1}(t)\right)\left(\theta(t)+\frac{\Lambda_{1}(t)}{p(t)}\right)\right] .
\end{gathered}
$$

Proof. It is easy to verify that problem (12) has a convex constrained set and a convex cost which is bounded below. These imply that (12) is a linearly constrained convex problem. Because problem (12) is feasible, it follows from Lagrange multiplier technique (see [28] for more details) that

$$
J^{*}=\max _{\lambda \in \mathbb{R}} \inf _{(\cdot), \pi(\cdot)) \text { is admissible }} J(\pi(\cdot), \lambda)<+\infty,
$$

where

$$
\begin{aligned}
J(\pi(\cdot), \lambda): & :=E(X(T)-c)^{2}+2 \lambda[E X(T)-c] \\
& =E(X(T)-c+\lambda)^{2}-\lambda^{2}
\end{aligned}
$$

For each fixed $\lambda$, the unconstrained problem

$$
J(\lambda):=\inf _{(X(\cdot), \pi(\cdot))} \text { is admissible } J(\pi(\cdot), \lambda)
$$

has the same form as (13). Then, it follows from Theorem 10 that

$$
\begin{aligned}
& J(\lambda)=-\lambda^{2}+p(0)\left(X_{0}-h(0)\right)^{2}+E \int_{0}^{T} p(t) \\
& \cdot\left[\left|\delta_{2}(t)-\eta_{2}(t)\right|^{2}+\left(\delta_{0}(t)-\eta_{0}(t)\right)^{2}\right] d t \\
& =E \int_{0}^{T} p(t)\left[\left|\delta_{2}(t)-(c-\lambda) q_{2}(t)-\bar{q}_{2}(t)\right|^{2}\right. \\
& \left.\quad+\left(\delta_{0}(t)-\bar{q}_{0}(t)\right)^{2}\right] d t-\lambda^{2}+p(0)\left[X_{0}-(c-\lambda)\right. \\
& \left.\cdot g_{1}(0)-g_{2}(0)\right]^{2},
\end{aligned}
$$


and the optimal investment strategy is

$$
\begin{aligned}
& \pi(t)=-\left(\sigma(t)^{-1}\right)^{\prime}\left[\delta_{1}(t)-\eta_{1}(t)\right. \\
& \left.+(X(t)-h(t))\left(\theta(t)+\frac{\Lambda_{1}(t)}{p(t)}\right)\right]=-\left(\sigma(t)^{-1}\right)^{\prime} \\
& \cdot\left[\delta_{1}(t)-(c-\lambda) q_{1}(t)\right. \\
& \left.-\bar{q}_{1}(t)\right]-\left(\sigma(t)^{-1}\right)^{\prime}\left[X(t)-(c-\lambda) g_{1}(t)\right. \\
& \left.-g_{2}(t)\right]\left(\theta(t)+\frac{\Lambda_{1}(t)}{p(t)}\right) .
\end{aligned}
$$

Rewriting $J(\lambda)$, we have

$$
\begin{aligned}
& J(\lambda)=(\lambda-c)^{2}\left(-1+p(0) g_{1}^{2}(0)\right. \\
& \left.+E \int_{0}^{T} p(t)\left|q_{2}(t)\right|^{2} d t\right)+2(\lambda-c)[-c \\
& +g_{1}(0) p(0)\left(X_{0}-g_{2}(0)\right) \\
& \left.+E \int_{0}^{T} p(t) q_{2}(t)^{\prime}\left(\delta_{2}(t)-\bar{q}_{2}(t)\right) d t\right]-c^{2} \\
& +p(0)\left(X_{0}-g_{2}(0)\right)^{2}+D=(\lambda-c)^{2}(-1 \\
& \left.+p(0) g_{1}^{2}(0)+M\right)+2(\lambda-c)[-c \\
& \left.+p(0) g_{1}(0)\left(X_{0}-g_{2}(0)\right)+k_{1}\right]-c^{2}+p(0)\left(X_{0}\right. \\
& \left.+g_{2}(0)\right)^{2}+D .
\end{aligned}
$$

Since $J(\lambda)$ is quadratic in $\lambda$ and $J^{*}$ is finite, we have

$$
1-M-p(0) g_{1}^{2}(0)>0
$$

In fact, if $1-M-p(0) g_{1}^{2}(0)=0$, then $J^{*}$ can only be finite when $-c+g_{1}(0) p(0)\left(X_{0}-g_{2}(0)\right)+k_{1}=0$ for any $c$, which is a contradiction. So it must be the case that $1-M-p(0) g_{1}^{2}(0)>$ 0 .

Rewriting (86), we have

$$
\begin{aligned}
J(\lambda)= & -\left(1-M-p(0) g_{1}^{2}(0)\right)(\lambda-c-k)^{2} \\
& +\left(1-M-p(0) g_{1}^{2}(0)\right) k^{2}-c^{2} \\
& +p(0)\left(X_{0}-g_{2}(0)\right)^{2}+D .
\end{aligned}
$$

Then we have the optimal $\lambda^{*}=c+k$ for (81). Taking $\lambda^{*}$ in (88) and (85),

$$
\begin{aligned}
& J^{*}=\left(1-M-p(0) g_{1}^{2}(0)\right) k^{2}-c^{2}+p(0)\left(X_{0}\right. \\
&\left.-g_{2}(0)\right)^{2}+D \\
&=\frac{\left[-c+k_{1}+p(0) g_{1}(0)\left(X_{0}-g_{2}(0)\right)\right]^{2}}{1-M-p(0) g_{1}^{2}(0)}-c^{2} \\
&+p(0)\left(X_{0}-g_{2}(0)\right)^{2}+D \\
&=\frac{M+p(0) g_{1}^{2}(0)}{1-M-p(0) g_{1}^{2}(0)}[c \\
&-\frac{\left.k_{1}+p(0) g_{1}(0)\left(X_{0}-g_{2}(0)\right)\right]^{2}+D+p(0)}{M+p(0) g_{1}^{2}(0)} \\
& \cdot\left(X_{0}-g_{2}(0)\right)^{2} \\
&-\frac{\left[k_{1}+p(0) g_{1}(0)\left(X_{0}-g_{2}(0)\right)\right]^{2}}{M+p(0) g_{1}^{2}(0)}, \\
&\left.+\left(X(t)+k g_{1}(t)-g_{2}(t)\right)\left(\theta(t)+\frac{\Lambda 1(t)}{p(t)}\right)\right] \\
& \pi^{*}(t)=-\left(\sigma(t)^{-1}\right)^{\prime}\left[\delta_{1}(t)+k q_{1}(t)-\bar{q}_{1}(t)\right.
\end{aligned}
$$

This completes the proof.

We claim that $D+p(0)\left(X_{0}-g_{2}(0)\right)^{2}-\left[k_{1}+p(0) g_{1}(0)\left(X_{0}-\right.\right.$ $\left.\left.g_{2}(0)\right)\right]^{2} /\left(M+p(0) g_{1}^{2}(0)\right) \geq 0$. In fact, since

$$
\begin{array}{r}
p(\cdot) \in \mathscr{L}_{\mathscr{F}}^{\infty}(\Omega ; C(0, T ; \mathbb{R})), \\
q_{2}(\cdot), \bar{q}_{2}(\cdot) \in \mathscr{L}_{\overparen{F}}^{2}\left(0, T ; \mathbb{R}^{d}\right), \\
\delta_{2}(\cdot) \in \mathscr{L}_{\overparen{F}}^{\infty}\left(0, T ; \mathbb{R}^{d}\right),
\end{array}
$$

we have $\sqrt{p(\cdot)}\left|\delta_{2}(\cdot)-\bar{q}_{2}(\cdot)\right|, \sqrt{p(\cdot)}\left|q_{2}(\cdot)\right| \in \mathscr{L}_{\overparen{F}}^{2}(0, T ; \mathbb{R})$. Further, because $\mathscr{L}_{\overparen{F}}^{2}(0, T ; \mathbb{R})$ is a Hilbert space, it follows from Cauchy-Schwarz's inequality that

$$
\begin{aligned}
& {\left[k_{1}+p(0) g_{1}(0)\left(X_{0}-g_{2}(0)\right)\right]^{2}=\left[E \int_{0}^{T} p(t) q_{2}(t)^{\prime}\right.} \\
& \left.\cdot\left(\delta_{2}(t)-\bar{q}_{2}(t)\right) d t\right]^{2}+\left[p ( 0 ) g _ { 1 } ( 0 ) \left(X_{0}\right.\right. \\
& \left.\left.\quad-g_{2}(0)\right)\right]^{2}+2 p(0) g_{1}(0)\left(X_{0}-g_{2}(0)\right) E \int_{0}^{T} p(t) \\
& \cdot q_{2}(t)^{\prime}\left(\delta_{2}(t)-\bar{q}_{2}(t)\right) d t \\
& \quad \leq\left[E \int_{0}^{T}\left(\sqrt{p(t)}\left|\delta_{2}(t)-\bar{q}_{2}(t)\right|\right)\right. \\
& \left.\cdot\left(\sqrt{p(t)}\left|q_{2}(t)\right|\right) d t\right]^{2}+\left[p ( 0 ) g _ { 1 } ( 0 ) \left(X_{0}\right.\right.
\end{aligned}
$$




$$
\begin{aligned}
& \left.\left.-g_{2}(0)\right)\right]^{2}+E \int_{0}^{T} 2\left(\sqrt{p(0) p(t)}\left|g_{1}(0)\right|\right. \\
& \left.\cdot\left|\delta_{2}(t)-\bar{q}_{2}(t)\right|\right)\left(\sqrt{p(0) p(t)}\left|X_{0}-g_{2}(0)\right|\right. \\
& \left.\cdot\left|q_{2}(t)\right|\right) d t \leq E \int_{0}^{T} p(t)\left|\delta_{2}(t)-\bar{q}_{2}(t)\right|^{2} d t \\
& \cdot E \int_{0}^{T} p(t)\left|q_{2}(t)\right|^{2} d t+\left[p ( 0 ) g _ { 1 } ( 0 ) \left(X_{0}\right.\right. \\
& \left.\left.-g_{2}(0)\right)\right]^{2}+E \int_{0}^{T} p(0) p(t)\left|g_{1}(0)\right|^{2} \mid \delta_{2}(t) \\
& -\left.\bar{q}_{2}(t)\right|^{2} d t+E \int_{0}^{T} p(0) p(t)\left|X_{0}-g_{2}(0)\right|^{2} \\
& \cdot\left|q_{2}(t)\right|^{2} d t,
\end{aligned}
$$

and so

$$
\begin{aligned}
& \left(M+p(0) g_{1}^{2}(0)\right)\left\{D+p(0)\left(X_{0}-g_{2}(0)\right)^{2}\right. \\
& \left.-\frac{\left[k_{1}+p(0) g_{1}(0)\left(X_{0}-g_{2}(0)\right)\right]^{2}}{M+p(0) g_{1}^{2}(0)}\right\}=p(0) \\
& \cdot g_{1}^{2}(0) E \int_{0}^{T} p(t) \\
& \cdot\left[\left|\delta_{2}(t)-\bar{q}_{2}(t)\right|^{2}+\left(\delta_{0}(t)-\bar{q}_{0}(t)\right)^{2}\right] d t \\
& +\left[p(0) g_{1}(0)\left(X_{0}-g_{2}(0)\right)\right]^{2}+p(0)\left(X_{0}\right. \\
& \left.-g_{2}(0)\right)^{2} E \int_{0}^{T} p(t)\left|q_{2}(t)\right|^{2} d t+E \int_{0}^{T} p(t) \\
& \cdot\left|\delta_{2}(t)-\bar{q}_{2}(t)\right|^{2} d t E \int_{0}^{T} p(t)\left|q_{2}(t)\right|^{2} d t \\
& +E \int_{0}^{T} p(t)\left(\delta_{0}(t)-\bar{q}_{0}(t)\right)^{2} d t E \int_{0}^{T} p(t) \\
& \cdot\left|q_{2}(t)\right|^{2} d t-\left[k_{1}+p(0) g_{1}(0)\left(X_{0}-g_{2}(0)\right)\right]^{2} \\
& \geq p(0) g_{1}^{2}(0) E \int_{0}^{T} p(t)\left(\delta_{0}(t)-\bar{q}_{0}(t)\right)^{2} d t \\
& +E \int_{0}^{T} p(t)\left(\delta_{0}(t)-\bar{q}_{0}(t)\right)^{2} d t E \int_{0}^{T} p(t) \\
& \left|q_{2}(t)\right|^{2} d t \geq 0
\end{aligned}
$$

Since $M+p(0) g_{1}^{2}(0)>0$, we have

$$
\begin{aligned}
D+ & p(0)\left(X_{0}-g_{2}(0)\right)^{2} \\
& -\frac{\left[k_{1}+p(0) g_{1}(0)\left(X_{0}-g_{2}(0)\right)\right]^{2}}{M+p(0) g_{1}^{2}(0)} \geq 0 .
\end{aligned}
$$

Remark 16. Theorem 15 shows that efficient frontier (79) is a parabola. Further, for a given mean target, the risk that the investor has to bear is given by (79). In particular, if the investor wants to take the global minimal risk, he/she can obtain the expected terminal wealth $\left(k_{1}+p(0) g_{1}(0)\left(X_{0}-\right.\right.$ $\left.\left.g_{2}(0)\right)\right) /\left(M+p(0) g_{1}^{2}(0)\right)$ by choosing the optimal strategy.

Remark 17. Theorem 15 also shows that the global minimal risk is

$$
\begin{aligned}
D & +p(0)\left(X_{0}-g_{2}(0)\right)^{2} \\
- & \frac{\left[k_{1}+p(0) g_{1}(0)\left(X_{0}-g_{2}(0)\right)\right]^{2}}{M+p(0) g_{1}^{2}(0)}
\end{aligned}
$$

which is nonnegative. This implies that when the market parameters are random and the financial market is incomplete, the liability can not be completely hedged.

Remark 18. Now we consider a financial market without liability; that is, $u(\cdot)=v(\cdot)=0$. Then, we have that $\delta(\cdot)=0$, $(0,0)$ is the unique solution of BSDE $(76)$ and the constants in Theorem 15 are given by

$$
\begin{aligned}
M & =E \int_{0}^{T} p(t)\left|q_{2}(t)\right|^{2} d t, \\
k_{1} & =0, \\
k & =\frac{-c+p(0) g_{1}(0) X_{0}}{1-M-p(0) g_{1}^{2}(0)}, \\
D & =0 .
\end{aligned}
$$

It follows from Theorem 15 that the efficient frontier in this case is given by

$$
\begin{aligned}
& \operatorname{Var} X^{*}(T) \\
& =\frac{M+p(0) g_{1}^{2}(0)}{1-M-p(0) g_{1}^{2}(0)}\left(c-\frac{p(0) g_{1}(0) X_{0}}{M+p(0) g_{1}^{2}(0)}\right)^{2} \\
& \quad+\frac{M p(0) X_{0}^{2}}{M+p(0) g_{1}^{2}(0)},
\end{aligned}
$$

which is the same as that of Lim [22]. This implies that Lim's result is a special case of our results. Therefore, our results generalize and improve Lim's results.

\section{Conclusions}

This paper studies the mean-variance asset-liability management problem with random market parameters. Since market parameters observed in the real world are always uncertain, it is more realistic to consider how to manage both assets and liabilities in a market with random market parameters. By using the theories of stochastic LQ control and BSDE, we derive both optimal investment strategies and the meanvariance efficient frontier. Compared with the existing results, the efficient frontier is still a parabola and liability does not 
affect the feasibility of the mean-variance portfolio selection problem in a complete market with random parameters. However, the liability can not be fully hedged in an incomplete market with random parameters.

Future studies can go one step further by considering this problem in a more complex market, whose prices are governed by SDEs with Lévy noise or Markovian switching. By using the methods and techniques proposed by Zhu $[29,30]$, it would be more interesting to discuss the optimal investment strategies and the efficient frontiers in the market mentioned above.

\section{Conflict of Interests}

The authors declare that there is no conflict of interests regarding the publication of this paper.

\section{Acknowledgments}

The authors are very grateful to the referee and the editor for patiently giving critical and insightful comments and suggestions, which helped to significantly improve the models, results, and presentation. The research was supported in part by National Natural Sciences Foundation of China under Grant 71571125, the Fundamental Research Funds for the Central Universities under Grant 2014SZYTD01, and Sichuan University under Grant 2014SCU04A06.

\section{References}

[1] H. Markowitz, "Portfolio selection," Journal of Finance, vol. 7, no. 1, pp. 77-91, 1952.

[2] S. Y. Wang and Y. S. Xia, Portfolio Selection and Asset Pricing, Springer, Berlin, Germany, 2002.

[3] D. Li and W.-L. Ng, "Optimal dynamic portfolio selection: multi-period mean-variance formulation," Mathematical Finance, vol. 10, no. 3, pp. 387-406, 2000.

[4] H. L. Wu and Z. F. Li, "Multi-period mean-variance portfolio selection with Markov regime switching and uncertain timehorizon," Journal of Systems Science and Complexity, vol. 24, no. 1, pp. 140-155, 2011.

[5] X. Y. Zhou and D. Li, "Continuous-time mean-variance portfolio selection: a stochastic LQ framework," Applied Mathematics and Optimization, vol. 42, no. 1, pp. 19-33, 2000.

[6] X. Li, X. Y. Zhou, and A. E. Lim, "Dynamic mean-variance portfolio selection with no-shorting constraints," SIAM Journal on Control and Optimization, vol. 40, no. 5, pp. 1540-1555, 2002.

[7] J. Xiong and X. Y. Zhou, "Mean-variance portfolio selection under partial information," SIAM Journal on Control and Optimization, vol. 46, no. 1, pp. 156-175, 2007.

[8] G. C. Wang and Z. Wu, "Mean-variance hedging and forwardbackward stochastic differential filtering equations," Abstract and Applied Analysis, vol. 2011, Article ID 310910, 20 pages, 2011.

[9] W. F. Sharpe and L. G. Tint, "Liabilities-a new approach," The Journal of Portfolio Management, vol. 16, no. 2, pp. 5-10, 1990.
[10] M. Leippold, F. Trojani, and P. Vanini, "A geometric approach to multiperiod mean variance optimization of assets and liabilities," Journal of Economic Dynamics \& Control, vol. 28, no. 6, pp. 1079-1113, 2004.

[11] M. C. Chiu and D. Li, "Asset and liability management under a continuous-time mean-variance optimization framework," Insurance: Mathematics \& Economics, vol. 39, no. 3, pp. 330-355, 2006.

[12] S. X. Xie, Z. F. Li, and S. Y. Wang, "Continuous-time portfolio selection with liability: meanvariance model and stochastic LQ approach," Insurance: Mathematics and Economics, vol. 42, no. 3, pp. 943-953, 2008.

[13] S. Xie, "Continuous-time mean-variance portfolio selection with liability and regime switching," Insurance: Mathematics \& Economics, vol. 45, no. 1, pp. 148-155, 2009.

[14] Y. Zeng and Z. F. Li, "Asset-liability management under benchmark and mean-variance criteria in a jump diffusion market," Journal of Systems Science and Complexity, vol. 24, no. 2, pp. 317327, 2011.

[15] H. Yao, Y. Lai, and Y. Li, "Continuous-time mean-variance assetliability management with endogenous liabilities," Insurance: Mathematics \& Economics, vol. 52, no. 1, pp. 6-17, 2013.

[16] J. Wei, K. C. Wong, S. C. Yam, and S. P. Yung, "Markowitz's mean-variance asset-liability management with regime switching: a time-consistent approach," Insurance: Mathematics \& Economics, vol. 53, no. 1, pp. 281-291, 2013.

[17] S. L. Heston, "A closed-form solution for options with stochastic volatility with applications to bond and currency options," The Review of Financial Studies, vol. 6, no. 2, pp. 327-343, 1993.

[18] J. Hull and A. White, "The pricing of options on assets with stochastic volatilities," Journal of Finance, vol. 42, no. 2, pp. 281$300,1987$.

[19] J. C. Cox and S. A. Ross, "The valuation of options for alternative stochastic processes," Journal of Financial Economics, vol. 3, no. 1-2, pp. 145-166, 1976.

[20] S. Beckers, "The constant elasticity of variance model and its implications for option pricing," Journal of Finance, vol. 35, no. 3, pp. 661-673, 1980.

[21] A. E. Lim and X. Y. Zhou, "Mean-variance portfolio selection with random parameters in a complete market," Mathematics of Operations Research, vol. 27, no. 1, pp. 101-120, 2002.

[22] A. E. B. Lim, "Quadratic hedging and mean-variance portfolio selection with random parameters in an incomplete market," Mathematics of Operations Research, vol. 29, no. 1, pp. 132-161, 2004.

[23] R. Norberg, "Ruin problems with assets and liabilities of diffusion type," Stochastic Processes and their Applications, vol. 81, no. 2, pp. 255-269, 1999.

[24] M. Decamps, A. de Schepper, and M. Goovaerts, "A path integral approach to asset-liability management," Physica A: Statistical Mechanics and its Applications, vol. 363, no. 2, pp. 404-416, 2006.

[25] H. K. Koo, "Consumption and portfolio selection with labor income: a continuous time approach," Mathematical Finance, vol. 8, no. 1, pp. 49-65, 1998.

[26] S. G. Peng, "A general stochastic maximum principle for optimal control problems," SIAM Journal on Control and Optimization, vol. 28, no. 4, pp. 966-979, 1990. 
[27] J. M. Yong and X. Y. Zhou, Stochastic Controls: Hamiltonian Syetems and HJB Equations, Springer, New York, NY, USA, 1999.

[28] D. G. Luenberger, Optimization by Vector Space Methods, John Wiley \& Sons, New York, NY, USA, 1968.

[29] Q. Zhu, "Asymptotic stability in the pth moment for stochastic differential equations with Lévy noise," Journal of Mathematical Analysis and Applications, vol. 416, no. 1, pp. 126-142, 2014.

[30] Q. X. Zhu, "Pth Moment exponential stability of impulsive stochastic functional differential equations with Markovian switching," Journal of the Franklin Institute, vol. 351, no. 7, pp. 3965-3986, 2014. 




Advances in

Operations Research

mansans



The Scientific World Journal
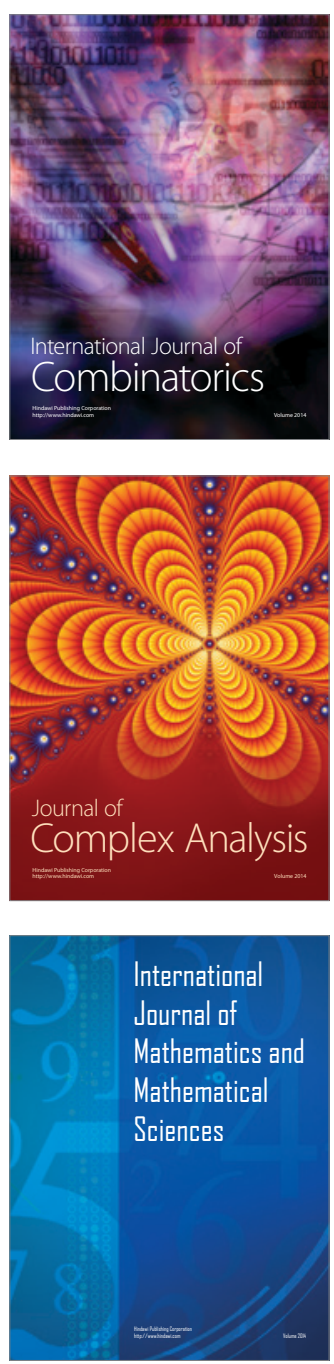


Submit your manuscripts at http://www.hindawi.com
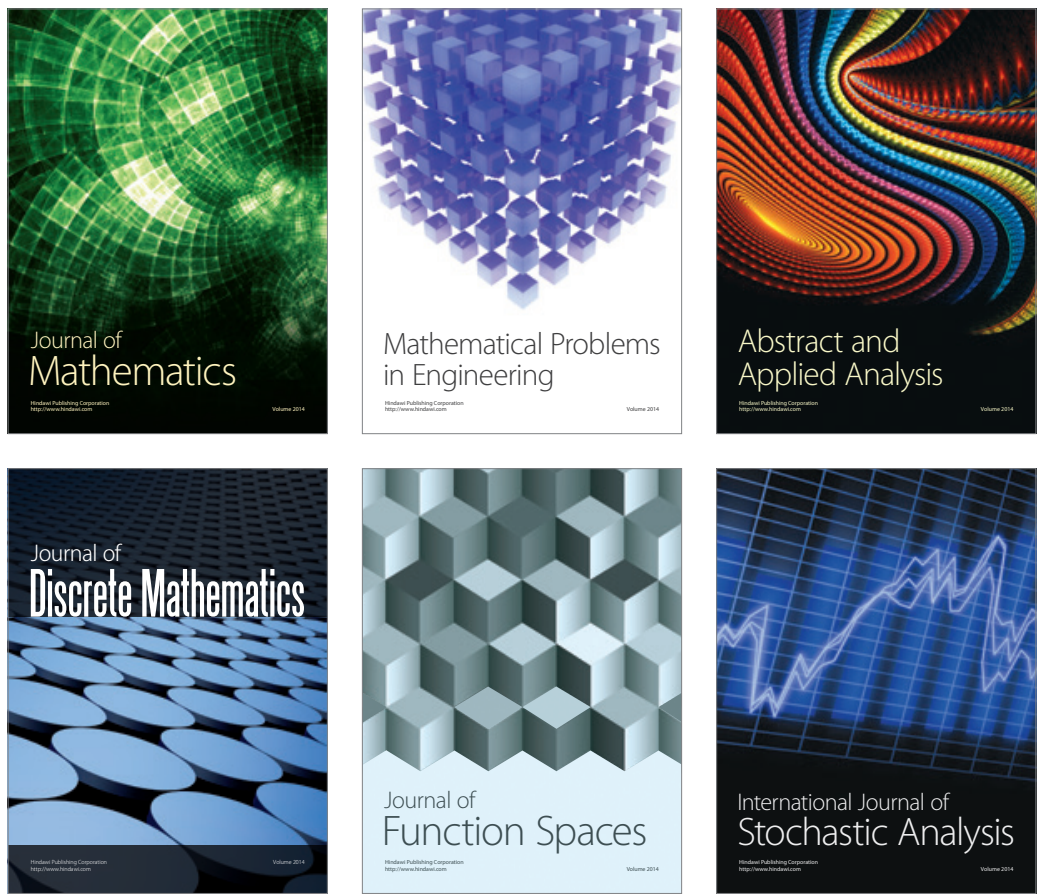

Journal of

Function Spaces

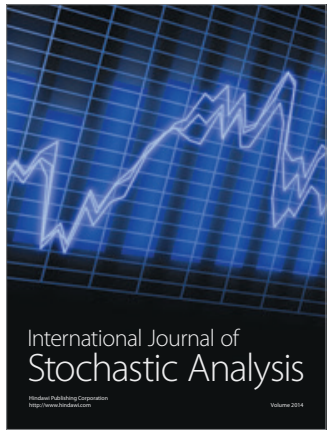

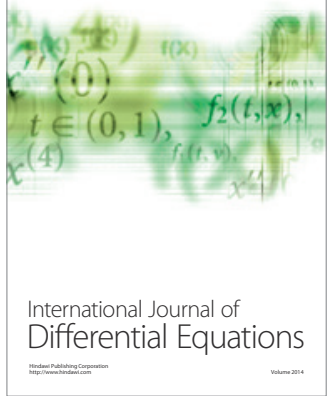
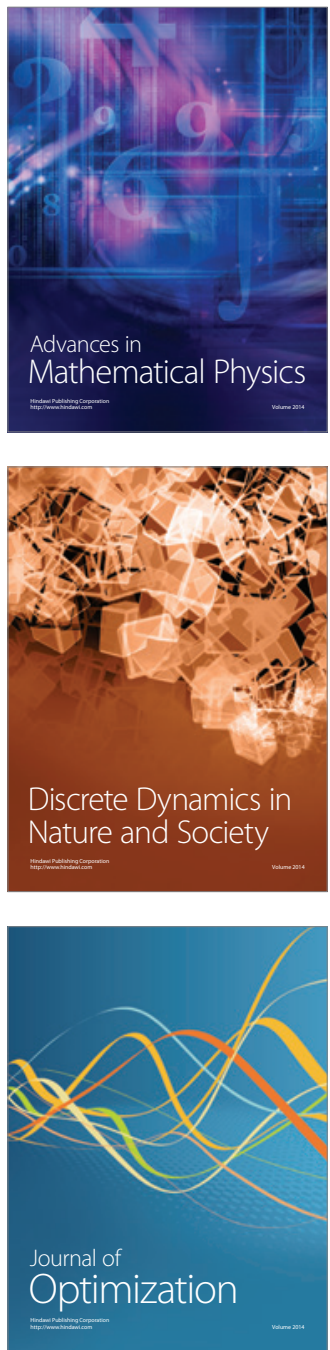